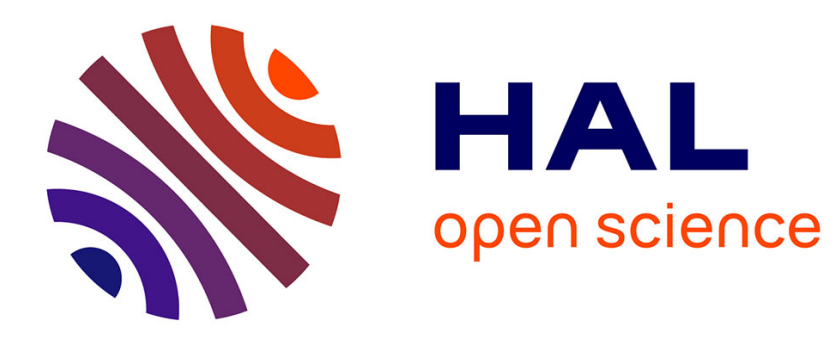

\title{
Les incitations à l'innovation dans le secteur privé
}

\author{
Claire Bonnard
}

\section{To cite this version:}

Claire Bonnard. Les incitations à l'innovation dans le secteur privé. 28èmes journées de microéconomie appliquée, Jun 2011, Sousse, Tunisie. halshs-00599700

\section{HAL Id: halshs-00599700 https://shs.hal.science/halshs-00599700}

Submitted on 10 Jun 2011

HAL is a multi-disciplinary open access archive for the deposit and dissemination of scientific research documents, whether they are published or not. The documents may come from teaching and research institutions in France or abroad, or from public or private research centers.
L'archive ouverte pluridisciplinaire HAL, est destinée au dépôt et à la diffusion de documents scientifiques de niveau recherche, publiés ou non, émanant des établissements d'enseignement et de recherche français ou étrangers, des laboratoires publics ou privés. 


\title{
$\underline{\text { Les incitations à l'innovation dans le secteur privé }}$
}

\author{
BONNARD Claire \\ IREDU-CNRS UMR 5225, University of Bourgogne \\ Pôle AAFE - Esplanade Erasme - B.P. 26513F - 21065 Dijon Cedex (France) \\ Tel: $00(0) 380395458$ \\ Fax: $00(0) 380395479$ \\ Mail: claire.bonnard@u-bourgogne.fr
}

\section{Résumé}

L'innovation est devenue un facteur clé de croissance économique. La question des incitations à l'innovation au sein des entreprises est donc primordiale. Dans ce papier, nous nous intéressons au type d'incitations monétaires reçues par les inventeurs au sein des entreprises avec une attention particulière à la mobilité inter-firme de ces derniers. Les résultats montrent un rendement salarial positif pour les inventeurs, celui-ci est plus important pour les inventeurs ayant connu une mobilité inter-firme, ce qui pourrait suggérer que les entreprises soient prêtes à payer les connaissances acquises par les inventeurs au sein des autres entreprises. Par contre, l'utilisation de stock-options comme incitation pour les inventeurs semble moins répandue dans les entreprises françaises que dans les entreprises étrangères.

\begin{abstract}
$\underline{\text { Abstract }}$
Innovation has become a key factor for economic growth. The issue of incentives as to boost innovation within firms is thus essential. In this paper, we are interested to discriminate the type of monetary incentives received by the inventors in firms ; with particular attention to inter-firm mobility of the professional. The results show a significant positive wage premium for the inventors, this one is more important for inventors who have experienced past mobility events, which might suggest that firms are willing to pay the specific knowledge gained by the inventors before in other firms. Additionally we show that incentive stock options for inventors seems less common in firms located in France than abroad.
\end{abstract}

Mot-clé: incitation, inventeur, score de propension

Keyword: incitation, inventor, propensity score

JEL: J33, O31, O32 


\section{Introduction}

L'innovation est devenue un facteur stratégique de croissance que ce soit au niveau des entreprises ou du pays. En effet, l'innovation est aujourd'hui considérée comme une des sources principales de croissance économique pour un pays (Jones, 2005). Au niveau des entreprises, l'innovation permet notamment de bénéficier d'un avantage compétitif. La plupart des études analysent l'innovation au niveau de l'entreprise. Pourtant, Rothaermel et Hess (2007) montrent que l'hétérogénéité des performances innovatrices entre les entreprises provient majoritairement des différences dans le capital humain intellectuel de celles-ci. Il est donc primordial de s'interroger sur les incitations à l'innovation au sein des entreprises. Les études récentes se sont plus particulièrement intéressées aux motivations des ingénieurs à inventer. Elles montrent que les inventeurs présentent une forte motivation intrinsèque, telles que le "challenge intellectuel" ou encore le "désir d'améliorer l'existence" (Sauerman et Cohen, 2008). Par contre, peu d'études ont analysé le type d'incitation mis en place pour les inventeurs au sein des entreprises. Tout d'abord, nous pouvons nous interroger sur la rentabilité pour un ingénieur de devenir inventeur ${ }^{1}$ ? D'autre part, les inventeurs connaissent une mobilité intra-firme importante (Traijtenberg, 2005). Une stratégie possible des entreprises est, en effet, d'attirer les inventeurs de leurs concurrents afin de bénéficier de leurs connaissances, de leurs savoirs. Les entreprises ont donc tout intérêt à mettre en place des incitations afin de retenir leurs chercheurs les plus productifs, plus globalement de protéger leur capital humain intellectuel.

Dans cet article, nous présentons, tout d'abord, quels types d'incitations ont été proposés dans la littérature économique, notamment dans le cadre de la théorie de l'agence et l'appliquons au cas spécifique des inventeurs. De la littérature ressort plus particulièrement deux types d'incitations monétaires pour les inventeurs qui sont le salaire et la détention de stock options. A partir des données de l'enquête du Conseil National des Ingénieurs et Scientifiques de France (CNISF), nous déterminons ensuite empiriquement le type d'incitation reçu par les inventeurs au sein des entreprises avec une attention particulière à la mobilité inter-firme de ces derniers.

\footnotetext{
${ }^{1}$ Nous appelons inventeur, tout ingénieur ayant déposé un brevet.
} 


\section{La théorie de l'agence appliquée aux inventeurs}

Les travailleurs de la connaissance sont la principale source d'innovation au sein des entreprises. La question de la productivité et des incitations des travailleurs de la connaissance est donc primordiale. Selon Osterloh (2005), cela entraîne un profond changement dans le management des ressources humaines. En effet, les ingénieurs et plus particulièrement les inventeurs présentent certaines spécificités qu'il s'agit de prendre en compte dans la mise en place d'incitation.

Tout d'abord, il est très difficile pour l'employeur de juger de l'effort fourni par l'ingénieur à innover. D'une part, observer le comportement de l'ingénieur offre peu d'information sur l'effort qu'il fournit puisque son travail est principalement cognitif. Par ailleurs, même s'il était observable, le manager peut difficilement apprécier si le comportement de l'ingénieur contribue ou non au succès de l'innovation (Zenger, 1994). En effet, les employés en R\&D sont fréquemment plus experts que les managers dans leur travail (Sauerman, Cohen, 2008). Il est, par conséquent, difficile pour l'employeur de mesurer la performance du chercheur. L'activité de recherche n'entraîne pas forcément des rendements immédiats (Lacetera et Zirulia, 2008). Un projet de recherche peut tout à fait échouer alors que le chercheur a fourni un effort important. Néanmoins, un indicateur possible de productivité peut être le fait de déposer un brevet, soit devenir inventeur.

D'autre part, les chercheurs ne développent pas uniquement des connaissances spécifiques à l'entreprise, mais également une forme de capital intellectuel qu'ils peuvent faire valorisés au sein d'autres entreprises (Gibbs, Levenson, 2002). Cet aléa moral sera d'autant plus important pour l'entreprise lorsque l'ingénieur devient inventeur. D'une part, les inventeurs peuvent être incités à exploiter leur résultat de recherche en rejoignant une entreprise concurrente (Moen, 2005). D'autre part, le brevet peut être perçu comme un signal des capacités et de productivité du scientifique (Toivanen et Väänänen, 2010). Les inventeurs peuvent donc faire valoriser leur portfolio de brevet sur le marché du travail et recevoir des offres de travail attractives de la part d'autres entreprises. La perte d'un chercheur productif peut donc s'avérer très coûteuse pour l'entreprise d'autant plus si l'inventeur rejoint une entreprise concurrente. Il semble que les inventeurs connaissent une mobilité inter-firme relativement importante. Dans l'étude de Traijtenberg (2005), 33\% des inventeurs américains ont connu une mobilité. 
Afin d'analyser quels types d'incitation peuvent être mis en place, la théorie de l'agence peut être mobilisée. Celle-ci s'applique à partir du moment où le principal et l'agent ont des désirs et des buts divergents et que le principal ne peut vérifier le comportement de l'agent (Eisenhardt, 1989). Dans le cas des inventeurs, l'employeur fait face à un premier aléa moral qui est l'observation de l'effort de l'employé, d'autant que selon Holmstrom (1979) plus la tâche demandée à l'employé concerne des connaissances très spécialisées, plus le hasard moral est significatif. Le second aléa moral correspond au risque de mobilité de l'inventeur dans une autre entreprise.

Dans le but de réduire l'aléa moral, deux solutions s'offrent au principal : contrôler le comportement de l'agent ou/et mettre en place des incitations pour que l'agent est le comportement désiré. Nous développons uniquement la seconde possibilité, soit l'instauration d'incitation. En effet, nous nous intéressons ici au personnel hautement qualifié. Or, lorsque le travail de l'agent demande une autonomie et une indépendance importantes ainsi que des compétences très spécialisées, il peut s'avérer difficile et coûteux pour le principal de contrôler le comportement de l'agent. Le principal aura donc tendance à mettre en place principalement des incitations pour que l'agent se conforme à ses exigences (Tosi et GomezMejia, 1989).

Dans cette section, nous présentons les différentes formes d'incitations proposées dans le cadre de la théorie principal-agent et les appliquons au cas particulier des inventeurs.

\subsection{Les incitations monétaires}

Les économistes se sont, tout d'abord, intéressés uniquement aux incitations monétaires. Selon la théorie de l'agence standard, l'effort peut être accru uniquement par des incitations monétaires. Ainsi, il est supposé que l'utilité de l'agent est une fonction croissante de sa richesse et qu'il présente une aversion au travail et plus particulièrement à l'effort (Holmstrom, 1979). Il s'agit donc pour le principal de trouver l'incitation monétaire la plus appropriée pour que l'agent adopte le comportement souhaité.

Selon la théorie du salaire d'efficience (Shapiro, Stiglitz, 1984), le principal peut être amené à offrir à l'agent un salaire supérieur au salaire de marché afin que ce dernier accroisse son niveau d'effort. Le principal va alors offrir un taux de salaire "efficient" pour lequel 
l'effort de l'agent est maximal. Ce modèle repose sur la "menace" puisque l'agent accroît son effort par peur d'être renvoyé et de ne pas retrouver le même niveau de salaire dans une autre entreprise. Salop (1979) montre que le principal peut également mettre en place un salaire plus élevé afin d'éviter le turnover, notamment lorsque les coûts d'embauche et de formations sont élevés. Dans le cas des inventeurs, les entreprises peuvent offrir un salaire plus élevé aux inventeurs les plus productifs pour qu'ils ne rejoignent pas une entreprise concurrente (Gersbach, Schmultzler, 2003). Par contre, selon Backer, Jensen et Murphy (1988), le fait que de plus hauts salaires permet de retenir l'agent s'explique facilement, par contre la relation entre plus haut salaire et effort de l'agent est moins claire. Ils montrent pour que l'agent accroisse son effort, le principal ne doit pas agir sur le niveau du salaire mais sur la forme de celui-ci.

Un autre système d'incitation proposé est la "paye à la performance", où le salaire est basé sur la performance, plus particulièrement sur la productivité de l'agent. Il est supposé que si les agents sont payés sur la base de leur performance, ils vont accroître leur effort et donc leur productivité, ce qui est nommé dans la littérature "effet prix" ou encore "effet disciplinaire". Analysant les contrats des chercheurs employés dans trois entreprises d'ingénierie électriques et chimiques en Allemagne depuis 1900, Burhop et Lübbers (2010) semblent confirmer cette théorie. En effet, les auteurs ne trouvent pas d'impact d'une augmentation de salaires sur la productivité des inventeurs. Il montrent, par contre, un impact positif lorsqu'il est versé aux inventeurs une part plus importante de bonus. Zenger et Lazzarini (2004) soulignent également que les systèmes de rémunération liés à la performance apparaissent plus efficaces pour motiver les ingénieurs à l'effort ainsi que pour attirer et retenir les ingénieurs "stars". Kochanski et Ledford (2001) notent que pour retenir les ingénieurs les plus productifs, il est nécessaire qu'ils perçoivent qu'ils sont traités différemment en terme salarial que les ingénieurs les moins productifs.

Giuri et al. (2007) montrent, par ailleurs, que, dans cinq pays européens (la France n'en fait pas parti), seulement $41.7 \%$ des inventeurs reçoivent une compensation monétaire et que celle-ci est dans la majorité des cas transitoire et non permanente. Le système de rémunération des inventeurs salariés varie grandement d'un pays à un autre. En France, selon la loi du 26 Novembre 1990, les entreprises ont l'obligation de verser une rétribution financière aux inventeurs salariés. Toutefois, le montant n'est pas fixé par la loi et est déterminé dans les conventions collectives, les accords d'entreprises et contrats individuels de travail. Une étude 
menée par l'Observatoire de la Propriété Intellectuelle en 2008 souligne néanmoins qu'il existe une grande hétérogénéité des rémunérations d'une entreprise à l'autre et qu'un système de rémunération n'est pas mis en place au sein de toutes les entreprises. Les compensations monétaires prennent principalement trois formes: des primes forfaitaires, une rémunération liée à l'exploitation ou encore une combinaison des deux. Le montant de ces dernières varient grandement. Selon cette étude, les primes forfaitaires peuvent varier d'un minimum médian de 500 à un maximum médian de 2000 euros.

Ce système de récompense a cependant fait l'objet de nombreuses critiques. Frey et Osterloh (2002) soulignent notamment que ce système de rémunération convient dans le cas de tâches routinières et concrètes et non dans le cas de tâches plus créatives et abstraites. Ce type d'incitation peut, par ailleurs, amener les agents à s'engager dans des tâches peu risquées et de court terme. D'autre part, la plupart des emplois particulièrement dans le cas de personnel hautement qualifié sont composés de plusieurs tâches complexes. Il est donc difficile pour le principal de mesurer objectivement la performance de l'agent. Les agents peuvent être incités à se focaliser uniquement sur la partie de l'emploi où ils sont récompensés au détriment des autres aspects de l'emploi (Prendergast, 1999). Ce type de système semble également inhiber la créativité des agents (Amabile, 1996).

D'autres incitations monétaires ont fait l'objet d'analyse telle que les incitations liées non plus à la performance de l'agent mais à la performance de l'entreprise tels que les stocks options. Contrairement, à la "paye à la performance", il s'agit ici d'incitations monétaires de long terme. La mise en place de stock-options s'est particulièrement développée ces cinq dernières années notamment dans le management des travailleurs hautement qualifiés (Mehran, Yermack, 1996, Lawler, 2003). Il semblerait que les incitations monétaires de long terme soient plus favorables à la motivation pour l'innovation (Ederer, Manso, 2009). Francis et al. (2009) soulignent qu'au vu de la nature de l'activité d'innovation, soit un niveau élevé de risque et une probabilité importante d'échec, l'incitation devrait être de long terme et protéger l'agent de l'échec. Dans leur étude, ils trouvent un lien positif entre les incitations par stockoptions et les indicateurs d'innovation tel que le nombre de brevet déposé par l'entreprise. Un résultat similaire est trouvé par Lerner et Wulf (2007). Analysant le lien entre innovation et compensations monétaires des responsables du département de R\&D mises en place au sein des entreprises, ils montrent que les incitations monétaires de long terme tels que les stocks options sont associées à des brevets plus fréquemment cités et de plus grande originalité. Lach 
et Schankerman (2008) montrent que les universités américaines générant le plus de revenu de licence sont celles où les chercheurs perçoivent une part plus importante de royalties.

Il apparaît que les stock-options puissent également être utilisées afin de sélectionner les meilleurs agents ou de les retenir au sein des entreprises (Oyer, Schaeffer, 2005). Les stock-options permettent de retenir les agents au sein des entreprises pour plusieurs raisons. D'une part, les entreprises imposent généralement un certain délai avant que l'employé puisse exercer ses stock-options ("vesting period"). Il s'agit d'accroître le coût de départ de l'entreprise de l'inventeur (Liebeskind, 1996). D'autre part, il est plus coûteux pour l'entreprise concurrente de recruter un agent détenteur de stock-options puisqu'elle va devoir compenser les gains que l'agent aurait pu percevoir sur le long terme grâce à celles-ci (Mehran, Yermack, 1996). Pakes et Nitzan (1983) analysent quels types de contrat peuvent être envisagés par les entreprises afin de retenir les inventeurs, de protéger leur niveau de capital intellectuel. Ils soulignent que les entreprises concurrentes sont généralement prêtes à offrir un salaire plus élevé afin de bénéficier des connaissances incorporées de l'inventeur. Ils montrent que les entreprises peuvent offrir des stock-options afin de les retenir.

Selon ces différentes théories, les contrats sont considérés comme statiques, simultanés. Des théories relâchent cette hypothèse, notamment celle du "career concern". Cette théorie initialement développée par Fama (1980) introduit les incitations implicites. En effet, dans ce cadre, les agents exercent des efforts non seulement pour maximiser leur salaire actuel mais également afin d'affecter la perception des autres (Prendergast, 1999), ce qui a été nommé "effet réputation". Ils ont implicitement une incitation à accroître leur niveau de réputation sur le marché du travail dans la perspective d'une meilleure carrière. Ce modèle semble plus particulièrement approprié pour les agents les moins expérimentés en début de carrière. Selon Fama (1980), par le mécanisme du marché, l'aléa moral disparaît dû aux incitations implicites des agents. Dans le cadre des ingénieurs, le dépôt de brevet permet à l'ingénieur d'accroître sa réputation sur le marché du travail et lui ouvre ainsi de nouvelles perspectives de carrière. Nous pouvons donc supposer que sans incitation explicite, l'ingénieur est tout de même implicitement incité à déposer un brevet d'autant qu'il existe une concurrence importante sur le marché du travail des ingénieurs et une forte mobilité interentreprise. Par contre, les entreprises ont tout intérêt à mettre en place des incitations afin que les inventeurs ne quittent pas l'entreprise pour une entreprise concurrente. Les entreprises 
peuvent faire signer un contrat de non concurrence. Or ce type de clause apparaît couteuse et est très encadrée.

\subsection{Motivation intrinsèque et incitation monétaire}

Néanmoins, les nouvelles théories, nommées théorie de l'agence comportementale montrent que toute incitation monétaire n'entraîne pas forcément un accroissement de l'effort et donc de la productivité de l'agent. Ceci provient du fait que contrairement à la théorie standard, la motivation intrinsèque des agents n'est plus considérée comme absente ou constante. Deci (1975) souligne qu'il est important de distinguer la motivation intrinsèque de la motivation extrinsèque des agents. La motivation extrinsèque correspond à toutes les incitations monétaires directes et indirectes et toutes formes de reconnaissance. En revanche, la motivation intrinsèque fait référence au désir de l'agent de se sentir compétent, de jouir d'une autonomie. Plus précisément, selon Frey et Osterloh (2002), la motivation intrinsèque des agents peut prendre principalement trois formes. Tout d'abord, l'individu peut retirer un plaisir, une satisfaction de l'activité elle-même. La seconde forme correspond au fait que l'activité exercée puisse correspondre aux valeurs, aux standards de l'agent tels que des valeurs éthiques. Enfin, la troisième forme est le plaisir de l'agent d'avoir achevé un but, d'avoir réussi un challenge. Contrairement à la motivation extrinsèque, la satisfaction retirée est ici immédiate. Les chercheurs semblent posséder une forte motivation intrinsèque. En effet, dès les années 50, la sociologie de la science montre l'existence d'une motivation intrinsèque importante de la part des chercheurs académiques. Merton (1973) indique que pour les chercheurs académiques, l'autonomie de satisfaire leur curiosité et la reconnaissance par leurs pairs sont des motivations plus importantes pour eux que de recevoir un salaire plus élevé. Des économistes (Levin, Stephan, 1991, Dasgupta, David, 1994) se sont également intéressés à ce sujet. Par exemple, Levin et Stephan (1991) signalent que les chercheurs académiques s'engagent dans la recherche pour deux raisons principales: la satisfaction de "résoudre des puzzles" et l'espérance de revenus futurs. Sauermann et Roach (2010) montrent que les docteurs se dirigeant vers le secteur industriel ont un goût pour la recherche moins prononcé que ceux s'orientant vers le secteur académique. En effet, ils semblent plus intéressés par le salaire ou encore l'accès aux ressources que les docteurs se dirigeant vers une 
carrière académique. D'après Stern (2004), les chercheurs dans le secteur industriel peuvent néanmoins présenter une motivation intrinsèque importante, ce qu'il a nommé "goût pour la recherche". La première étude présentant la motivation intrinsèque des inventeurs est celle de Rossman datant de 1931. En interrogeant 700 déclarants de brevets sur leur motivation à inventer, l'auteur montre que les deux premiers motifs évoqués sont "l'amour de la science" et "le désir d'améliorer l'existence", le salaire n'apparaissant qu'en troisième position. Dans une étude plus récente, Giuri et al. (2007) trouvent des résultats semblables. Dans six pays européens, les motivations à inventer déclarées par les ingénieurs sont dans une plus grande mesure des motivations personnelles (satisfaction personnelle) et sociales (prestige, réputation) que des motivations monétaires.

Dans ce cadre d'analyse, la motivation intrinsèque peut donc influer sur le niveau d'effort des agents (Frey et Jegen, 2001). Cela change radicalement avec la théorie standard dans le sens où l'effort est susceptible d'accroître l'utilité de l'agent, il n'est plus supposé exister une complète "désutilité de l'effort" (Kreps, 1997). D'autre part, la motivation extrinsèque et intrinsèque ne sont plus supposées être indépendantes l'une de l'autre. Elles ne sont également pas additives ; il existe, au contraire, une relation dynamique systématique entre les deux, nommé "crowding effect" (Frey et Osterloh., 2002) . Il est possible que la mise en place d'incitation extrinsèque entraîne une diminution de la motivation intrinsèque des agents, "effet d'éviction" ("crowd out") ou, au contraire, accroit la motivation intrinsèque des agents, "effet d'agglomération" ("crowd in") (Frey, Jegen, 2001, Bénabou , Tirole, 2003). Frey et Jegen (2001) soulignent qu'un "effet d'éviction" est susceptible d'apparaître lorsque par la mise en place d'incitation extrinsèque, l'agent se sent contrôlé ; à l'inverse l'"effet d'agglomération" apparaîtra quand l'agent se sent reconnu, soutenu. Des applications empiriques semblent confirmer l'existence de tels effets dans plusieurs domaines (cf. Frey et Jegen, 2001). Dans le cadre de l'innovation, Sauerman et Cohen (2008) trouvent que les inventeurs présentant une motivation plus importante pour le challenge dans leur travail ou les responsabilités fournissent des efforts supérieurs dans leur travail (mesurés en nombre d'heure travaillé), par rapport aux ingénieurs évoquant d'autres motifs de motivation. Par contre, les inventeurs déclarant avoir une motivation pour le challenge et dans une moindre mesure pour le salaire semblent être plus productifs que les autres inventeurs.

Selon cette théorie ("crowd theory"), les incitations monétaires ne sont donc pas forcément plus efficientes que toute autre forme d'incitation. En effet, les incitations non 
monétaires peuvent s'avérer être des instruments efficaces. Dans certaines situations, il est supposé que l'instauration d'incitations non monétaires entraîne un "effet d'éviction" moins important sur la motivation intrinsèque des agents que les incitations monétaires. Des études (Radhakrishnan, Ronen, 1999 ; Frey, 2007) montrent bien que les incitations non monétaires telles que les "prix", le "challenge", la "gratification" permettent d'accroître l'effort de l'agent, et peuvent même s'avérer plus efficaces que les incitations monétaires. L'enquête de l'INPI de 2008 révèle que des entreprises mettent en place des programmes de reconnaissance tels que des lettres de félicitation ou encore une remise de prix. Par exemple, l'entreprise Air Liquide en plus d'une compensation monétaire versée aux inventeurs organise annuellement une cérémonie pour distinguer les meilleures inventions.

Au vu des ces différentes études, il semble que les inventeurs soient motivés par deux types de motivations, extrinsèque et intrinsèque. Il s'agit pour l'employeur de déterminer le type d'incitations approprié permettant de concilier ces deux types de motivations. Ces différents types de motivation pourraient expliquer pourquoi les incitations mises en place pour l'innovation diffèrent notamment selon la nature de l'entreprise. Honig-Haftel et Martin (1993) soulignent que les ingénieurs dans les grandes entreprises semblent avoir une motivation intrinsèque plus importante que les ingénieurs dans les petites entreprises. Les premiers seraient plus motivés par des incitations non monétaires que par des incitations monétaires, contrairement aux ingénieurs au sein des petites entreprises qui ne réagissent qu'aux incitations monétaires.

Peu d'étude empirique ont analysé la rémunération de l'innovation au sein des entreprises privées. A notre connaissance, les seules recherches empiriques analysant la rémunération des inventeurs sont celles de Toivanen et Väänänen (2010) en Finlande et celle de Harhoff et Hoisl (2007) en Allemagne. Ces études trouvent, en moyenne, un avantage salarial pour les inventeurs. En effet, Toivanen et Väänänen (2010) montrent que les inventeurs perçoivent en moyenne un gain salarial de 3\% l'année après avoir déposé un brevet et bénéficient d'un accroissement de salaire de 4 à $5 \%$ trois à quatre ans après. Ils soulignent également que ce rendement varie de façon importante en fonction de la qualité du brevet. Par contre, ils ne trouvent pas de rendement salarial de la mobilité pour les inventeurs. L'étude de Harhoff et Hoisl (2007) s'intéresse plus particulièrement à l'impact de la législation sur la 
rémunération des inventeurs. Ils trouvent des résultats similaires à l'étude précédente. Il semblerait que la compensation monétaire des inventeurs dépende principalement de la qualité du brevet déposé.

Dans cet article, nous nous intéressons plus précisément aux incitations monétaires à l'innovation au sein du secteur privé. Nous nous interrogeons sur la rentabilité pour un ingénieur de devenir inventeur. De ces différentes études, principalement deux types d'incitations monétaires pour les inventeurs semblent se dégager, le salaire et la détention de stock options. Dans la suite de ce papier, nous analysons empiriquement ces deux formes de compensation monétaire avec une attention particulière à la mobilité de l'inventeur et à la nature de son entreprise. Nous nous intéressons également aux ingénieurs travaillant à l'étranger afin d'analyser dans quelle mesure le type de compensation reçue par ces inventeurs peut différer par rapport à celle reçue par les inventeurs français.

\section{La méthodologie utilisée: Méthode par appariement}

Dans cette étude, nous cherchons à analyser le rendement salarial ainsi que la détention de stock-options des inventeurs. La difficulté provient de l'existence de biais de sélection. Il est fort probable que les inventeurs et non inventeurs différent dans leurs caractéristiques individuelles et de leur entreprise. Les études montrent, en effet, que la productivité des chercheurs peut être fortement influencer par des facteurs individuels ou encore organisationnels. Il est donc important de prendre en compte cette hétérogénéité.

Dans ce papier, nous utilisons l'approche par appariement ("matching") initiée par Rosenbaum et Rubin (1983). Ces méthodes ont été utilisées principalement pour l'évaluation des politiques publiques. Récemment, ces méthodes ont également été appliquées dans l'étude des écarts salariaux entre les hommes et les femmes (Frölich, 2007) ou encore dans le rendement de l'enseignement supérieur (Titus, 2007).

L'idée de la méthode par appariement est d'associer à chaque individu traité (ici, les inventeurs) un individu non traité (les non-inventeurs), dont les caractéristiques sont identiques à celles de l'individu traité (Brodaty et $a l .$, 2007). Sous certaines hypothèses, nous 
pouvons ensuite déterminer l'effet moyen du traitement dans la population des individus traités (ATT), qui correspond dans note étude à l'avantage salarial des inventeurs.

Soit $D_{i}=1$ pour les ingénieurs ayant déposé un brevet, ici les "inventeurs", et $D_{i}=0$ les ingénieurs n'ayant pas déposé de brevet. $Y_{i}$ représente la variable d'intérêt, dans notre étude le salaire annuel des ingénieurs. L'effet de traitement, ici devenir inventeur est égal à $\Delta_{i}=Y_{i 1}-$ $Y_{i 0}$. Or, il n'est pas possible d'observer un même individu face aux deux situations de traitement, nous devons donc mesurer l'effet moyen sur la population traitée (les inventeurs) que nous notons :

$$
\Delta_{A T T}=E\left(Y_{i 1} \mid D_{i}=1\right)-E\left(Y_{i 0} \mid D_{i}=1\right)
$$

Nous cherchons à estimer l'impact moyen du traitement sur les traités nommé ici ATT ("Average Treatment effect on the Treated").

Nous ne pouvons, par ailleurs, estimer $E\left(Y_{i 0} \mid D_{i}=1\right)$. Nous connaissons $E\left(Y_{i 0} \mid D_{i}=0\right)$ mais nous ne pouvons pas nous en servir comme substitut. En effet, il est fort probable du fait de notre hypothèse que les variables déterminants la décision de traitement (devenir inventeur) détermine également la variable d'intérêt (le salaire). Par conséquent, le revenu des individus traités et des individus non traités différeront même en absence de traitement, ceci correspond au biais de sélection (Caliendo, Kopeinig., 2008).

On pourra donc estimer $\Delta_{A T T}$ qu'à la condition que $E\left(Y_{i 0} \mid D_{i}=1\right)-E\left(Y_{i 0} \mid D_{i}=0\right)=0$

Pour cela, l'indépendance conditionnelle aux caractéristiques observables (Conditional Independance Assumption) doit être supposée. Selon cette hypothèse, la variable d'intérêt doit être indépendante au fait d'être traité contrôlé par les variables $X$ décrivant l'individu. Cette hypothèse sous entend donc que la sélection est basée uniquement sur les caractéristiques observées $X$ et que l'ensemble des variables influençant simultanément le traitement et la variable de résultat sont observés par le chercheur (Caliendo, Kopeinig, 2008). Formellement, cette hypothèse s'écrit :

$$
Y_{i 1}, Y_{i 0} \sqcup D \mid X, \forall X
$$

ou encore $E\left(Y_{i 0} \mid X, D=1\right)=E\left(Y_{i 0} \mid X, D=0\right)$

Nous pouvons écrire sous cette hypothèse: 


$$
E\left(Y_{i 1} \mid D=1\right)-E\left(Y_{i 0} \mid D=1\right)=E_{x \mid D=1}\left\{E\left(Y_{i} \mid X, D=1\right)-E\left(Y_{i} \mid X, D=0\right)\right\}
$$

Le principe de l'estimation consiste à utiliser les informations dont on dispose sur les individus non traités pour construire pour chaque individu traité un contrefactuel, c'est à dire une estimation de ce qu'aurait été sa situation s'il n'avait pas été traité (Brodaty et al., 2007). Cette hypothèse suppose néanmoins la prise en compte d'un nombre important de variables $X$, l'appariement peut donc s'avérer difficile.

Rosenbaum et Rubin (1983) proposent une méthode alternative au problème de dimension du vecteur $X$. Il s'agit de la méthode d'appariement sur score de propension. Ce score correspond à la probabilité, pour une personne de caractéristiques $X$ données, d'être exposé au traitement. Ils montrent, en effet, que la propriété d'indépendance en relation des caractères observés $X$ implique celle d'indépendance à la variable indiquant la probabilité de traitement, ou score de propension à être traité (Brodaty et al., 2007) noté $P(X)=\operatorname{Pr}(D=$ $1 \mid X)$.

Formellement :

$$
Y_{i 1}, Y_{i 0} \sqcup D \mid P(X), \forall X
$$

L'appariement peut donc maintenant s'écrire :

$$
\Delta_{A T T}=E_{P(x \mid) D=1}\left\{E\left(Y_{i} \mid D=1, P(X)\right)-E\left(Y_{i} \mid D=0, P(X)\right)\right\}
$$

Pour la méthode d'appariement, une seconde hypothèse importante nommée support commun (Common support) doit être respectée. Cette hypothèse impose que l'on dispose pour chaque individu traité, d'individus non traités dont les scores ont des valeurs proches du score de l'individu traité (Brodaty et al., 2007). Cette hypothèse permet de s'assurer que pour chaque unité traitée, il y a des unités de contrôle s'appuyant sur les mêmes variables observées X. Formellement :

$$
0<\operatorname{Pr}(D=1 \mid X)<1
$$

Dans un premier temps, il s'agit d'estimer le score de propension. En principe, tout modèle à choix discret peut être utilisé (Caliendo, Kopeinig, 2008). Dans ce papier, nous choisissons un modèle Probit puisque notre variable s'assimile à la situation ou non d'être inventeur. Le choix des variables pour l'estimation du score de propension est très importante 
puisque pour respecter l'hypothèse du CIA, la variable d'intérêt doit être indépendante au fait d'être traité conditionnellement au score de propension. Dans la section suivante, nous justifions plus précisément le choix des variables sélectionnées pour notre étude.

Dans un second temps, nous effectuons l'appariement. Il existe plusieurs méthodes d'estimation, Smith et Todd (2005) soulignent que les différentes méthodes d'appariement entraînent généralement des résultats similaires lorsqu'on dispose d'une base de données importante. Puisqu'il n'existe pas de consensus dans la littérature sur la meilleure méthode d'estimation, nous utilisons différentes techniques qui sont l'estimation par le plus proche voisin ("nearest neighbor"), avec remise n des plus proches voisins et avec noyaux ("kernel").

La méthode la plus usuelle est celle du plus proche voisin. Cette méthode consiste à retenir pour chaque individu traité, l'individu non traité avec le score de propension le plus proche du sien. La principale limite de cette méthode est qu'elle peut effectuer un appariement entre deux individus avec une distance en terme de score de propension assez importante. Il est alors possible que de mauvais appariements soient effectués. Une façon de remédier à cette limite est d'effecteur l'appariement à un seuil donné en imposant une distance maximale entre les scores de propension. D'autre part, la méthode par le plus proche voisin peut s'effecteur sans remise (l'individu du groupe de contrôle n'est sélectionné qu'une seule fois dans la construction du contrefactuel) ou avec remise (l'individu peut être sélectionné plusieurs fois). Il est également possible de retenir plusieurs voisins, ceci en fonction d'une zone d'acceptation du voisinage possible, ce qu'on nomme la méthode par les plus proches voisins.

Une autre méthode d'estimation possible est celle avec noyaux développée par Heckman, Ichimura et Todd (1997, 1998). Celle-ci utilise des moyennes pondérées de l'ensemble des individus du groupe de contrôle pour construire le contrefactuel. Il s'agit de retenir, pour chaque individu traité, tous les individus non traités, mais en les affectant d'un poids inversement proportionnel à leur "distance" avec l'individu traité. Frölich (2007) souligne que c'est cette méthode qui donne les résultats les plus précis et ceci d'autant plus que la distance est mesurée de manière fiable dans le processus.

La principale limite de l'approche par appariement est qu'elle prend en compte uniquement l'hétérogénéité observable. Or, des caractéristiques non observables, telle que la motivation peuvent biaiser les résultats. De ce fait, l'existence de caractéristiques non 
observables violerait l'hypothèse CIA, la sélection ne s'opérerait plus uniquement sur les caractéristiques observables. Dans la littérature économique, plusieurs méthodes ont été développées afin de prendre en compte l'existence de caractéristiques non observables. Les méthodes habituellement utilisées sont les approches classiques de correction des biais en "deux étapes" tels que le modèle d'Heckman et les régressions instrumentales. La principale difficulté de ces méthodes est de trouver des variables instrumentales valides. En effet, il s'agit de trouver au moins une variable qui affecte le fait de déposer un brevet mais qui n'a pas d'impact sur le salaire. Nous ne disposons directement pas de ce type de variables dans notre base de données. Néanmoins, Rosenbaum (2002) a développé un test nous permettant de tester la robustesse de nos estimations par rapport à l'hétérogénéité non observée.

La probabilité de bénéficier du traitement pour un individu i avec des caractéristiques observables $x_{i}$ peut s'écrire : $P\left(x_{i}\right)=P\left(D_{i}=1 \mid x_{i}\right)=F\left(\beta x_{i}+\gamma u_{i}\right)$ où $u_{i}$ est une variable non observable et $\gamma$ l'effet de $u_{i}$ sur le fait de bénéficier du traitement. La probabilité de participation ne dépendra uniquement de $x_{i}$ seulement si $\gamma$ est égal à zéro. Par conséquent, si deux individus $i, j$ ont les mêmes caractéristiques $X$, alors leur probabilité $P\left(x_{i}\right)$ et $P\left(x_{j}\right)$ devrait être égal. Nous parlerons de "biais cachés" (hidden bias) lorsque deux individus $i, j$ présentant les mêmes caractéristiques $\left(x_{i}=x_{j}\right)$ ont des probabilités différentes de traitement $\left(P\left(x_{i}\right) \neq P\left(x_{j}\right)\right)$. Afin de simplifier, Aakvik (2001) suppose que la variable non observable $u_{i}$ est dichotomique. Il prend l'exemple de la motivation qui est un déterminant à la fois de la participation et de la variable d'intérêt, la personne est motivée $(u=1)$ ou pas $(u=0)$. Nous pouvons dès lors écrire le rapport de cote (odds ratio) pour deux individus $i$ et $j$ :

$$
\frac{1}{\Gamma} \leq \frac{\left(P_{i}\right)\left(1-\left(P_{j}\right)\right)}{\left(P_{j}\right)\left(1-\left(P_{i}\right)\right)} \leq \Gamma
$$

La méthode de Rosenbaum (2002) repose sur la sensibilité du paramètre $\Gamma$. Si $\lceil=1$, les individus avec les mêmes caractéristiques $X$ ont la même probabilité de traitement, il n'existe pas de "biais cachés". Par contre, si deux individus identiques ont un 「 égal, par exemple, à 2, cela signifie qu'un des deux individus peut avoir une probabilité deux fois supérieure par rapport à l'autre de recevoir le traitement. Ce résultat peut s'expliquer par le fait que ces deux individus différent par leurs caractéristiques non observables (Rosenbaum, 2002). Il s'agit ainsi de faire varier l'influence Ґ et de vérifier si les conclusions de l'analyse statistique peuvent être invalidées. Dans cet article, nous faisons varier 「 de 1 à 2 par tranches de 0,05. 


\section{Les données}

Les données utilisées proviennent d'une étude conduite chaque année (tous les 2 ans jusqu'en 2002) par le CNISF (Conseil National des Ingénieurs et des Scientifiques de France) auprès des diplômés des écoles d'ingénieurs, quels que soient leur âge et leur expérience. Environ 47.500 ingénieurs répondent habituellement au questionnaire. Cet article utilise les données de l'étude conduite en 2009, portant sur la situation des ingénieurs au 31 décembre 2008. Pour chaque enquête, un thème particulier est analysé plus précisément. Pour la 20ème enquête, le CNISF s'est penché sur les questions de l'innovation au sein des entreprises. Ces données nous permettent d'avoir les informations nécessaires sur les caractéristiques individuelles et de l'entreprise des inventeurs.

Seuls les ingénieurs travaillant dans le secteur marchand sont ici pris en compte, ceux employés par les administrations, les universités et les laboratoires publics sont exclus de l'analyse. Nous avons un échantillon d'environ 16000 ingénieurs.

\subsection{La mesure du brevet}

La variable dépendante concernant les brevets est issue de l'item suivant : Dans les 5 dernières années, avez-vous été déposant ou co-déposant inventeur ou inventeur salarié d'un brevet?

Nous sommes conscients que cette variable comporte plusieurs limites pour la mesure de la productivité de l'ingénieur. Mais, nous ne disposons pas d'informations sur la commercialisation du brevet déposé et dans quelle mesure celui-ci a entraîné un profit à l'entreprise de l'inventeur. Giuri et al. (2007) soulignent, toutefois, que la plupart des brevets n'entraîne pas de succès commercial. A partir de nos données, nous ne pouvons connaître la valeur, la "qualité" du brevet. Les études utilisent habituellement comme mesure de "qualité" du brevet, le nombre de citations reçus par le brevet déposé. Nous n'avons pas de telle donnée. Le fait qu'on se situe sur la période des cinq dernières années entraîne également certains biais. Cette variable ne nous permet notamment pas de mesurer le gain salarial des inventeurs sur le long terme. Or, comme nous l'avons souligné auparavant, la rémunération des brevets peut différer dans le temps, d'autant plus que la valeur du brevet est, en moyenne, révélée au bout de trois années. 


\subsection{La mesure de la mobilité}

D'autre part, les ingénieurs reportent les caractéristiques de leur entreprise uniquement à la date de l'enquête. Cependant, il est possible que lors des cinq dernières années de l'enquête, les ingénieurs aient changé d'entreprise. Nous estimons donc les mêmes modèles sur les deux sous échantillons, les ingénieurs déclarant n'avoir pas connu de mobilité ces cinq dernières années et ceux ayant connu une mobilité. Les ingénieurs n'ayant pas connu de mobilité représente $60 \%$ de notre échantillon contre $40 \%$ des ingénieurs ayant connu une mobilité. Nous ne savons donc pas si l'ingénieur a changé d'entreprise quand il est devenu inventeur ou s'il est devenu inventeur une fois qu'il a changé d'entreprise. Néanmoins, Traijtenberg (2005) et Hoisl (2007) montrent qu'il existe une relation simultanée entre mobilité et productivité des inventeurs. Il semble, en effet, que lorsque l'inventeur connait une mobilité, il devient plus productif. Hoisl (2007) explique ce résultat par un meilleur appariement entre l'employé et l'employeur et/ou l'inventeur profite des connaissances de ces nouveaux collègues. En même temps, Traijtenberg (2005) montre que les inventeurs les plus productifs ex ante ont plus de chances de connaître une mobilité. Dans les deux cas, la mobilité d'un tel ingénieur entraîne un coût non négligeable pour l'entreprise.

\subsection{Les expatriés}

Dans notre échantillon, 13,6\% des ingénieurs travaillent à l'étranger. Parmi ceux-ci, environ $60 \%$ travaillent dans un pays d'Europe autre que la France (12\% en Suisse, $14 \%$ en Allemagne, 6\% en Belgique et 9\% en Grande Bretagne). Parmi les 40\% restants, 20\% travaillent aux Etats-Unis. Dans notre échantillon, les inventeurs travaillant à l'étranger représentent $12 \%$ de l'ensemble des inventeurs. Nous sommes néanmoins conscients qu'il est susceptible d'exister certains biais dû notamment aux non-réponses.

\subsection{Sélection des variables}

Afin d'estimer le score de propension, le choix des variables est très important. Caliendo et al. (2008) soulignent, d'ailleurs, que le fait d'omettre d'importantes variables pour l'analyse risque d'entraîner des résultats biaisés. Il est recommandé de choisir les variables sur la base des théories économiques et des études empiriques antérieures. 
Tout d'abord, les incitations à innover pour l'ingénieur seront différentes en fonction de certaines caractéristiques individuelles de l'ingénieur. Nous retenons comme caractéristiques individuelles : l'ancienneté (Mariani, Romanelli, 2007, Hoisl, 2007, Toivanen et Väänänen, 2010), le niveau de capital humain (Mariani et al., 2007) ainsi que le genre (Giuri et al., 2007, Whittington, Smith Doer, 2005).

Il est également nécessaire de s'intéresser aux caractéristiques de l'emploi et de l'entreprise de l'ingénieur. Au niveau de l'emploi de l'ingénieur, nous introduisons les variables concernant l'activité dominante de l'ingénieur et plus particulièrement si l'activité principale de l'ingénieur concerne l'étude, la recherche et la conception. Nous prenons également en compte le fait que l'ingénieur a participé à un programme de R\&D de l'Union Européenne ou non. Les caractéristiques de l'entreprise prises en considération sont la taille de l'entreprise (Hoisl, 2007, Kim et al., 2004, Mariani et al., 2007), le secteur de l'entreprise et l'intensité en terme d'innovation de l'entreprise (Balkin et Gomez-Mejia, 1984). Afin de mesurer les différences d'intensité d'innovation entre les entreprises, nous prenons en considérations trois variables qui sont le niveau de dépense interne de R\&D de l'entreprise (Griliches, 1984, Crépon et al., 1998, Mairesse, Mohnen, 2005), la mise en place par l'entreprise d'une politique de veille concurrentielle stratégique et enfin l'existence de partenariat avec des universités (George et al., 2002). 
Tableau $\mathrm{n}^{\circ}$ : Statistiques descriptives

\begin{tabular}{|c|c|c|c|c|}
\hline$(\mathrm{en} \%)$ & Inventeurs & Non-inventeurs & Ensemble & Sign. $^{2}$ \\
\hline Caractéristiques individuelles & & & & \\
\hline Genre & $\begin{array}{l}89,9 \\
10,1\end{array}$ & $\begin{array}{l}85,7 \\
14,3\end{array}$ & $\begin{array}{l}86,2 \\
13,8\end{array}$ & $* * *$ \\
\hline Doctorat & 18,7 & 3,8 & 5,4 & $* * *$ \\
\hline $\begin{array}{l}\text { Ancienneté sur le marché du travail } \\
\text { Moyenne }\end{array}$ & 10,52 & 9,28 & 9,41 & $* * *$ \\
\hline $\begin{array}{l}\text { Activité dominante de l'ingénieur } \\
\text { Recherche, Etude, Essais } \\
\text { Autres activités }\end{array}$ & $\begin{array}{l}75,7 \\
24,3\end{array}$ & $\begin{array}{l}35,4 \\
64,6\end{array}$ & $\begin{array}{l}39,8 \\
60,2\end{array}$ & $* * *$ \\
\hline Participation à un projet de l'UE & 35,6 & 9,7 & 12,5 & $* * *$ \\
\hline Caractéristiques de l'entreprise & & & & \\
\hline $\begin{array}{l}\text { Secteur de l'entreprise } \\
\text { - Minerais, métallurgie, fonderie, travail des métaux } \\
\text {-Production minéraux non métalliques } \\
\text {-Industrie chimique } \\
\text { - Fabrication d'équipements mécaniques, de machines } \\
\text { - Matériel électrique, électronique, informatique } \\
\text { - Constructions automobiles, navales } \\
\text {-Industrie textile } \\
\text {-Papier carton } \\
\text { - Autre industrie } \\
\text { - Autres secteurs }\end{array}$ & $\begin{array}{c}3,83 \\
2,70 \\
5,87 \\
8,71 \\
14,71 \\
18,54 \\
0,73 \\
2,37 \\
6,13 \\
36,41\end{array}$ & $\begin{array}{c}2,58 \\
1,19 \\
3,78 \\
4,55 \\
8,62 \\
10,13 \\
0,46 \\
1,43 \\
2,80 \\
57,9\end{array}$ & $\begin{array}{c}2,71 \\
1,36 \\
4,01 \\
5,01 \\
9,29 \\
11,05 \\
0,49 \\
1,53 \\
3,17 \\
61,38\end{array}$ & $* * *$ \\
\hline $\begin{array}{l}\text { Taille de l'entreprise } \\
\qquad \begin{array}{r}\text { Moins de } 2000 \text { salariés } \\
\text { Plus de } 2000\end{array}\end{array}$ & $\begin{array}{l}39,9 \\
60,1\end{array}$ & $\begin{array}{l}47,3 \\
52,7\end{array}$ & $\begin{array}{l}46,5 \\
53,5\end{array}$ & $* * *$ \\
\hline $\begin{array}{r}\text { Politique de veille concurrentielle stratégique } \\
\text { Oui } \\
\text { Non } \\
\text { Ne sait pas }\end{array}$ & $\begin{array}{c}83,9 \\
9,6 \\
6,5\end{array}$ & $\begin{array}{l}66,8 \\
17,8 \\
15,4\end{array}$ & $\begin{array}{l}68,7 \\
16,9 \\
14,4\end{array}$ & $* * *$ \\
\hline $\begin{array}{l}\text { \% du CA en dépenses de R\&D } \\
\text { Entre } 0 \text { et } 5 \% \\
\text { Plus de } 5 \%\end{array}$ & $\begin{array}{l}19,4 \\
52,3 \\
28,3\end{array}$ & $\begin{array}{l}20,1 \\
29,4 \\
50,5\end{array}$ & $\begin{array}{l}21 \\
31 \\
48\end{array}$ & $* * *$ \\
\hline Partenariat avec des universités & $\begin{array}{l}70,5 \\
18,2 \\
11,3\end{array}$ & $\begin{array}{l}44,3 \\
29,0 \\
26,7\end{array}$ & $\begin{array}{c}47,2 \\
27,8 \\
25\end{array}$ & $* * *$ \\
\hline
\end{tabular}

Note:*** significatif à $1 \%, * *$ significatif à 5\%, * significatif à 10

Les statistiques descriptives sont présentées dans le tableau $\mathrm{n}^{\circ} 1$. Tout d'abord, les inventeurs et non inventeurs présentent des caractéristiques individuelles significativement différentes. En effet, 10,1\% des inventeurs sont des femmes contre $14 \%$ parmi les noninventeurs. Les inventeurs possèdent plus fréquemment un doctorat que les non-inventeurs $(18,7 \%$ contre $3,8 \%)$. Ils ont également en moyenne une ancienneté sur le marché du travail plus importante que les non inventeurs (10,52 ans contre 9,28 ans). D'autre part, les inventeurs travaillent plus fréquemment dans des activités de R\&D (76\% contre $35 \%)$ et ont participé plus fréquemment à un programme de R\&D de l'UE (36\% contre 10\%) par rapport aux non-inventeurs. Au niveau des caractéristiques de l'entreprise, il existe également une différence significative entre les deux types d'ingénieur. Il semblerait que les inventeurs soient

\footnotetext{
${ }^{2}$ Test de student pour les variables quantitatives et test de Chi-2 pour les variables qualitatives.
} 
employés plus fréquemment dans des entreprises de taille plus importante. Le tableau $\mathrm{n}^{\circ} 1$ montre également une différence importante dans la stratégie de leur entreprise en termes d'innovation. Les inventeurs se trouvent plus fréquemment dans des entreprises effectuant une politique de veille stratégique $(83,9 \%$ contre $66,8 \%)$ et ayant des dépenses de R\&D internes importantes (52,3\% contre 29,4\%). Enfin, une part beaucoup plus importante d'inventeurs travaille dans une entreprise collaborant avec des universités par rapport aux noninventeurs $(70,5 \%$ contre $44,3 \%)$.

\section{Estimation du score de propension}

\subsection{Qualité de l'estimation}

Dans un premier temps, le score de propension a été estimé à l'aide d'un probit. Avant de présenter les résultats, il est important de vérifier la qualité de l'estimation du score de propension. Afin de juger celle-ci, il est nécessaire de vérifier la propriété d'"équilibrage" ainsi que de déterminer le support commun. La propriété d'"équilibrage" est généralement vérifiée par deux tests qui sont le test d'égalité des moyennes et le test des différences standardisées, tous deux développés par Rosenbaum et Rubin (1985). Les résultats sont présentés dans le tableau $1 \mathrm{~A}$ en annexe $\mathrm{A}$.

Avant appariement, nous avons vu que les différences de caractéristiques $X$ sont significatives entre les inventeurs et non inventeurs. Si l'appariement est correct, il ne devrait donc plus avoir de différence significative de moyenne entre les deux groupes d'individus, c'est le principe du test d'égalité de moyenne. Le test des différences standardisées permet, quant à lui, de déterminer la réduction du biais dû à l'appariement par le score de propension. Rosenbaum et Rubin (1985) soulignent qu'une différence standardisée supérieure à 20 devrait être considérée comme trop importante. Après appariement, les différences de caractéristiques deviennent non significatives sauf pour l'ancienneté (significatif à 10\%) et l'activité dominante de l'ingénieur (significatif à 10\%). Concernant les différences standardisées, aucune valeur n'est supérieure à $|20|$. Au vu de ces tests, la qualité du score de propension semble correcte. 
Afin de déterminer le support commun, nous imposons la condition du "minimamaxima". Cette condition consiste à enlever les observations de la population traitée (les inventeurs) dont le score de propension est plus élevé que le maximum et plus faible que le minimum du score de propension de la population de comparaison (les non-inventeurs). Les graphiques de score de propension sont présentés en annexe A. Le respect de cette hypothèse enlève moins de $1 \%$ de nos observations.

\subsection{Devenir inventeur}

Les résultats du probit concernant la probabilité de devenir inventeur sont présentés en annexe dans le tableau 1B. Les estimations ont été effectuées sur l'ensemble de l'échantillon et sur les deux sous ensembles comprenant uniquement les ingénieurs n'ayant pas connu de mobilité et les ingénieurs ayant connu une mobilité ces cinq dernières années. Les résultats différent très peu selon l'échantillon pris en compte.

Les résultats sont similaires à ceux trouvés dans la littérature. En effet, les femmes ont une probabilité significativement plus faible de déposer un brevet par rapport aux hommes. Nous pouvons tout de même souligner que les études (McMillan, 2009, Whittington , Smith Doer, 2005) montrent que les brevets déposés par les femmes sont en terme de qualité (mesurée par le nombre de citation) similaire voire supérieure à ceux déposés par les hommes. Il semble également exister une relation croissante à taux décroissant entre la productivité et l'ancienneté de l'ingénieur. En effet, la variable ancienneté est significativement positive alors que son carré est négatif. D'autre part, être docteur accroît fortement les chances de déposer un brevet. Il semble donc que les docteurs soient plus productifs en R\&D. Résultat non surprenant, le fait d'être employé dans des activités d'étude, de recherche et de conception accroît les chances de déposer un brevet et les ingénieurs ayant participé ces cinq dernières années à un programme de R\&D de l'UE ont une chance significativement plus élevée de devenir inventeur.

Les caractéristiques de l'entreprise de l'ingénieur semblent également influer sur le fait de devenir inventeur. L'ensemble des variables décrivant la stratégie de l'entreprise en termes d'innovation sont significatives. La probabilité de déposer un brevet pour un ingénieur ne semble pas être statistiquement significative lorsqu'il est employé dans une entreprise avec des dépenses internes de $R \& D$ supérieures à $5 \%$. Le fait que l'entreprise de l'ingénieur exerce 
une politique de veille concurrentielle stratégique a un impact positif significatif. Il semblerait que la collaboration entre l'entreprise et les universités soit également un facteur très significatif sur la productivité en terme de R\&D.

Par ailleurs, les ingénieurs ne connaissant pas le montant des dépenses de R\&D de leur entreprise et si celle-ci collabore avec des universités ont une probabilité plus faible de devenir inventeur. Nous pouvons supposer que les inventeurs sont plus intéressés par la politique d'innovation de leur entreprise que les non-inventeurs. Il semble aussi que le fait d'être employé dans une entreprise de grande taille (mesurée en nombre d'employés) influe significativement sur le fait de devenir inventeur.

\section{Les incitations monétaires des inventeurs}

Après avoir déterminé le score de propension, nous pouvons estimé d'une part, l'avantage salarial pour les inventeurs et d'autre part, la détention de stock-options. Les estimations sont effectuées uniquement sur la population des docteurs-ingénieurs. Caliendo et al. (2008) soulignent, en effet, que si certaines variables nous semblent importantes dans le traitement, il peut s'avérer intéressant de faire les analyses sur ces sous-groupes. Or, nous avons vu auparavant que les docteurs représentent une part importante d'inventeurs, environ $20 \%$.

\subsection{L'avantage salarial des inventeurs}

Dans un premier temps, nous effectuons un appariement afin d'estimer l'avantage salarial pour les inventeurs. Le tableau ci-dessous présente les résultats selon les différentes méthodes d'estimation utilisées. Le tableau 2 présente deux estimateurs qui sont l'estimateur naïf et l'effet moyen global (ATT). L'estimateur naïf correspond à la différence de moyenne des salaires entre les deux groupes d'ingénieurs sans prise en compte des caractéristiques $X$. L'ATT est l'estimateur qui nous intéresse, il correspond à l'estimateur après appariement. Nous présentons également les résultats des MCO afin de comparer les résultats. 
Tableau $\mathrm{n}^{\circ} 2$ : Résultats par la méthode par appariement

\begin{tabular}{|c|c|c|c|c|}
\hline & & $\begin{array}{c}\text { Plus proche } \\
\text { voisin } \\
\text { Caliper }^{3} 0,01 \\
\text { Avec remise }\end{array}$ & $\begin{array}{l}\text { Plus proches voisins avec remise } \\
\qquad n=5\end{array}$ & Noyau \\
\hline \multirow{4}{*}{ Ensemble des ingénieurs ${ }^{4}$} & Estimateur naif & \multicolumn{3}{|c|}{$0,071 * * *$} \\
\hline & $\mathrm{MCO}^{5}$ & \multicolumn{3}{|c|}{$0,029 * * *$} \\
\hline & ATT & $0,04 * *$ & $0,034 * * *$ & $0,029 * * *$ \\
\hline & $\begin{array}{l}\text { Support } \\
\text { commun }\end{array}$ & 13776 & 13776 & 13779 \\
\hline \multirow{4}{*}{ Ingénieurs sans mobilité } & Estimateur naïf & \multicolumn{3}{|c|}{$0,084 * * *$} \\
\hline & $\mathrm{MCO}$ & \multicolumn{3}{|c|}{$0,027 * * *$} \\
\hline & ATT & $0,036^{*}$ & $0,033^{* * *}$ & $0,027 * *$ \\
\hline & $\begin{array}{l}\text { Support } \\
\text { commun }\end{array}$ & 8446 & 8446 & 8455 \\
\hline \multirow{4}{*}{ Ingénieurs avec mobilité } & Estimateur naïf & \multicolumn{3}{|c|}{$0,058 * * *$} \\
\hline & $\mathrm{MCO}$ & \multicolumn{3}{|c|}{$0,036^{* * * *}$} \\
\hline & ATT & $0,059 * *$ & $0,050 * *$ & $0,033 * *$ \\
\hline & $\begin{array}{l}\text { Support } \\
\text { commun }\end{array}$ & 5318 & 5318 & 5324 \\
\hline \multirow{4}{*}{ Docteurs } & Estimateur naïf & \multicolumn{3}{|c|}{$0,067 * *$} \\
\hline & $\mathrm{MCO}$ & \multicolumn{3}{|c|}{$0,061 * * *$} \\
\hline & ATT & $0,088 * *$ & $0,086^{* * *}$ & 0,063 *** \\
\hline & $\begin{array}{l}\text { Support } \\
\text { commun }\end{array}$ & 731 & 731 & 733 \\
\hline \multirow{4}{*}{ Entreprise de - de 500 salariés } & Estimateur naïf & \multicolumn{3}{|c|}{$0,124 * * *$} \\
\hline & $\mathrm{MCO}$ & \multicolumn{3}{|c|}{$0,052 * * *$} \\
\hline & ATT & $0,10 * * *$ & $0,063 * * *$ & $0,069 * * *$ \\
\hline & $\begin{array}{l}\text { Support } \\
\text { commun }\end{array}$ & 4474 & 4474 & 4477 \\
\hline \multirow{4}{*}{$\begin{array}{l}\text { Entreprise de + de } 500 \\
\text { salariés }\end{array}$} & Estimateur naïf & \multicolumn{3}{|c|}{$0,038 * * *$} \\
\hline & $\mathrm{MCO}$ & \multicolumn{3}{|c|}{$0,02 * *$} \\
\hline & ATT & $0,036^{*}$ & 0,022 & $0,016^{*}$ \\
\hline & $\begin{array}{c}\text { Support } \\
\text { commun }\end{array}$ & 9301 & 9301 & 9301 \\
\hline \multirow{4}{*}{ "PME de haute technologie ${ }^{6 "}$} & Estimateur naïf & \multicolumn{3}{|c|}{$0,082 * * *$} \\
\hline & $\mathrm{MCO}$ & & $0,063 * * *$ & \\
\hline & ATT & $0,195 * * *$ & 0,153 *** & $0,107 * * *$ \\
\hline & $\begin{array}{l}\text { Support } \\
\text { commun }\end{array}$ & 1172 & 1172 & 1176 \\
\hline
\end{tabular}

Note: L'écart-type des estimations est obtenu par la méthode des bootstrap (1000 réplications)

Tout d'abord, les estimateurs naïfs sont positifs et significatifs pour l'ensemble des échantillons. Les résultats des estimations après appariement montrent un gain salarial positif significatif pour les inventeurs quelque soit l'échantillon utilisé. Nous pouvons noter que pour l'ensemble des échantillons, les estimations par le plus proche voisin sont supérieures aux autres estimations. Ceci provient du fait que ce type d'estimation est moins précis puisque le "plus proche voisin" peut correspondre à un mauvais appariement. Pour l'ensemble des ingénieurs, l'estimation par le plus proche voisin est de $4,0 \%$ alors que les estimations par les

\footnotetext{
${ }^{3}$ Caliper (ou compas) est une norme permettant de définir la zone de voisinage.

${ }^{4}$ Nous nous intéressons ici uniquement aux ingénieurs travaillant en France.

${ }^{5}$ Les estimations détaillées des MCO sont présentées en annexe dans le tableau 1C.

${ }^{6}$ Nous définissons les PME de haute technologie, les entreprises de moins de 500 salariés avec une part de Chiffre d'Affaire consacrée à la R\&D supérieure de 5\% ou déposant un brevet chaque année.
} 
plus proches voisins et avec noyaux sont respectivement de $3,4 \%$ et de $2,9 \%$, significatifs à 1\%. Par ailleurs, il semble que le rendement de l'innovation soit plus important pour les docteurs. En effet, l'effet moyen global est de 8,8\% par le plus proche voisin et de 6,3\% par noyaux.

Il apparaît que les ingénieurs ayant connu une mobilité présentent un gain salarial à l'innovation supérieur aux ingénieurs n'ayant pas connu de mobilité. L'estimation respectivement par les plus proches voisins et par noyaux montre un rendement salarial de l'ordre de $5 \%$ et $3,3 \%$ pour les premiers contre $3,3 \%$ et $2,7 \%$ pour les ingénieurs sans mobilité. Il est vrai que nous ne savons pas si l'ingénieur a changé d'entreprise quand il est devenu inventeur ou s'il est devenu inventeur une fois qu'il a changé d'entreprise. Néanmoins, comme nous l'avons souligné auparavant, il semble exister une relation simultanée entre mobilité et productivité des inventeurs (Traijtenberg, (2005) et Hoisl (2007)). Ce résultat semble tout de même suggérer que les entreprises payent les connaissances acquises par les ingénieurs au sein des autres entreprises (Dahl, 2002).

D'autre part, le rendement salarial de l'innovation semble dépendre également du type d'entreprise où se trouve l'inventeur. En effet, dans les entreprises de moins de 500 salariés, les inventeurs perçoivent un avantage salarial d'environ 6,9\% (estimateur par noyaux), alors que celui-ci est de seulement 1,6\% pour les inventeurs employés dans une grande entreprise. Ce résultat pourrait s'expliquer par, comme nous l'avons évoqué précédemment, une motivation intrinsèque différente des inventeurs selon la taille de l'entreprise. Au sein des entreprises, nommées ici PME de haute technologique, l'innovation apparaît fortement valorisée avec un rendement salarial pour les inventeurs supérieur à 10\% (significatif à 1\%).

Enfin, les résultats des $\mathrm{MCO}$ montrent également un coefficient positif et significatif du fait de déposer un brevet. L'ordre de grandeur est sensiblement le même que les estimations par appariement. Nous pouvons, par ailleurs, souligner que le fait de travailler dans des activités de $R \& D$ entraine une moindre rémunération significative pour les ingénieurs (tableau 1C). En effet, les ingénieurs travaillant dans le secteur R\&D /Conception ont un salaire inférieur de 8,5\%, l'écart salarial est de 10,6\% (significatif à 1\%) pour les docteurs-ingénieurs. Des études antérieures (Bonnard, Boudon, Paul, 2011) ont trouvé des résultats similaires. Ces résultats laissent supposer qu'au sein des entreprises, l'innovation est rentable pour les ingénieurs qu'en termes de résultat, de performance. 


\subsection{La détention de stock-options}

Le tableau $\mathrm{n}^{\circ} 3$ montre que les inventeurs perçoivent une part plus importante de stocks options que les ingénieurs non inventeurs. 5,0\% des inventeurs ont perçu des stocks options en 2008 contre 3,7\% des non inventeurs. La différence est plus prononcée pour les ingénieurs n'ayant pas connu de mobilité inter-firme ces cinq dernières avec 5,0\% des inventeurs possédant des stocks options contre 3,3\% des non-inventeurs.

Tableau n³: Statistiques descriptives: Détention de Stocks Options

\begin{tabular}{|c|c|c|c|c|c|c|c|c|c|}
\hline & \multicolumn{3}{|c|}{ Ensemble } & \multicolumn{3}{c|}{ Ingénieurs sans mobilité } & \multicolumn{3}{c|}{ Ingénieurs avec mobilité } \\
\hline & Inventeurs & Non Inven & Total & Inventeurs & Non Inven & Total & Inventeurs & Non Inven & Total \\
\hline Stock Options & 5,0 & 3,7 & 3,8 & 4,9 & 3,3 & 3,5 & 5,1 & 4,3 & 4,4 \\
\hline Non stocks options & 95,0 & 96,3 & 96,2 & 95,1 & 96,7 & 96,5 & 94,9 & 95,7 & 95,6 \\
\hline Total & 100 & 100 & 100 & 100 & 100 & 100 & 100 & 100 & 100 \\
\hline
\end{tabular}

Nous effectuons l'appariement avec la détention de stock-options comme variable d'intérêt. Les résultats sont présentés dans le tableau $n^{\circ} 4$.

Tableau n 4 : Résultats de la méthode par appariement: Détenir des stock-options.

\begin{tabular}{|c|c|c|c|c|}
\hline & & $\begin{array}{l}\text { Plus proche } \\
\text { voisin } \\
\text { Caliper } 0,01 \\
\text { Avec remise }\end{array}$ & $\begin{array}{l}\text { Plus proches voisins avec } \\
\text { remise } n=5\end{array}$ & Noyau \\
\hline \multirow{3}{*}{ Ensemble des ingénieurs } & Estimateur naïf & \multicolumn{3}{|c|}{$0,013 * * *$} \\
\hline & ATT & 0,015 & 0,01 & 0,01 \\
\hline & $\begin{array}{l}\text { Support } \\
\text { commun }\end{array}$ & 13776 & 13776 & 13779 \\
\hline \multirow{3}{*}{ Ingénieurs sans mobilité } & Estimateur naïf & \multicolumn{3}{|c|}{$0,016 * * *$} \\
\hline & ATT & $0,027 * *$ & 0,012 & $0,014 *$ \\
\hline & $\begin{array}{l}\text { Support } \\
\text { commun }\end{array}$ & 8446 & 8446 & 8445 \\
\hline \multirow{3}{*}{ Ingénieurs avec mobilité } & Estimateur naïf & \multicolumn{3}{|c|}{0,01} \\
\hline & ATT & 0,01 & 0,00 & $-0,00$ \\
\hline & $\begin{array}{c}\text { Support } \\
\text { commun }\end{array}$ & 5318 & 5318 & 5324 \\
\hline \multirow{3}{*}{$\begin{array}{l}\text { Entreprise moins de } 500 \\
\text { salariés }\end{array}$} & Estimateur naïf & \multicolumn{3}{|c|}{$0,026 * * *$} \\
\hline & ATT & 0,00 & 0,011 & 0,01 \\
\hline & $\begin{array}{l}\text { Support } \\
\text { commun }\end{array}$ & 4474 & 4474 & 4477 \\
\hline \multirow{3}{*}{$\begin{array}{l}\text { Entreprise plus de } 500 \\
\text { salariés }\end{array}$} & Estimateur naïf & \multicolumn{3}{|c|}{0,01} \\
\hline & ATT & $0,026 * *$ & 0,01 & 0,01 \\
\hline & $\begin{array}{l}\text { Support } \\
\text { commun }\end{array}$ & 9301 & 9301 & 9301 \\
\hline
\end{tabular}

Note: L'écart-type des estimations est obtenu par la méthode des bootstrap (1000 réplications)

Pour l'ensemble des ingénieurs, l'ATT est positif et non significatif quelque soit la méthode utilisée. Par contre, les résultats sont différents si l'on prend en compte uniquement 
les ingénieurs ayant connu une mobilité ou les ingénieurs n'ayant pas connu de mobilité. En effet, l'effet global moyen est positif et significatif de l'ordre de 1,5\% pour les ingénieurs n'ayant pas connu de mobilité alors qu'il est non significatif pour les ingénieurs ayant connu une mobilité. Ces résultats suggèrent que les entreprises puissent offrir des stocks-options aux inventeurs afin qu'ils ne quittent pas l'entreprise. Ils sont en concordance avec la littérature soulignant que les incitations par stock-options peut être plus particulièrement utilisé afin d'éviter le turnover et de retenir les employés au sein de l'entreprise (Oyer et Schaefer, 2005).

Il ne semble, par ailleurs, pas exister des différences selon la taille de l'entreprise. L'ATT est non significatif quelque soit la taille de l'entreprise.

\subsection{Le cas des expatriés}

Nous nous intéressons ici plus particulièrement aux ingénieurs travaillant à l'étranger. Cette analyse nous permet ainsi de déterminer s'il existe un système d'incitation pour les inventeurs différent dans les entreprises étrangères que dans les entreprises françaises. Les résultats sont reportés dans le tableau $n^{\circ} 5$ ci-dessous.

Tableau $n^{\circ} 5$ : Résultats des estimations pour le sous-échantillon des ingénieurs expatriés

\begin{tabular}{|c|c|c|c|c|}
\hline Variable d'intérêt & & $\begin{array}{c}\text { Plus proche } \\
\text { voisin } \\
\text { Caliper } 0,01 \\
\text { Avec remise }\end{array}$ & $\begin{array}{l}\text { Plus proches voisins avec } \\
\text { remise } n=5\end{array}$ & Noyau \\
\hline \multirow{4}{*}{ Salaire } & Estimateur naïf & \multicolumn{3}{|c|}{$0,069 * *$} \\
\hline & MCO & \multicolumn{3}{|c|}{$-0,01$} \\
\hline & ATT & 0,048 & 0,029 & 0,024 \\
\hline & $\begin{array}{l}\text { Support } \\
\text { commun }\end{array}$ & 2169 & 2169 & 2174 \\
\hline \multirow{3}{*}{ Stock-options } & Estimateur naïf & \multicolumn{3}{|c|}{$0,118 * * *$} \\
\hline & ATT & $0,146^{* * *}$ & $0,118 * * *$ & $0,129 * * *$ \\
\hline & $\begin{array}{l}\text { Support } \\
\text { commun }\end{array}$ & 2169 & 2169 & 2174 \\
\hline \multirow{3}{*}{$\begin{array}{l}\text { Attribution gratuite } \\
\text { d'action }\end{array}$} & Estimateur naïf & \multicolumn{3}{|c|}{$0,03 *$} \\
\hline & ATT & $0,061 * *$ & $0,064 * *$ & $0,054 * *$ \\
\hline & $\begin{array}{l}\text { Support } \\
\text { commun }\end{array}$ & 2169 & 2169 & 2174 \\
\hline
\end{tabular}

Pour les ingénieurs travaillant à l'étranger, nous trouvons des résultats très différents à ceux trouvés en France. En effet, il ne semble pas que les inventeurs à l'étranger perçoivent une rémunération significativement supérieure par rapport aux ingénieurs non-inventeurs. En revanche, la détention de stock-options ou l'attribution gratuite d'action sont fortement 
significatives pour les inventeurs à l'étranger. Plusieurs explications peuvent expliquer ce résultat. Dans la littérature, il est souligné que les entreprises peuvent offrir des stock-options afin d'attirer les ingénieurs les plus talentueux ("stars") (Hall, Murphy, 2003). Toutefois, en France, l'accès aux stock-options semble plus particulièrement lié à la position hiérarchique de l'individu (Mendez, 2006) et le traitement fiscal sur les stock-options et l'attribution gratuite d'action apparaît plus contraignante que dans d'autres pays européens tels qu'en Grande Bretagne ou encore aux Etats-Unis (Andreosso-O'Callaghan, 2000).

\section{Qualité de l'appariement et robustesse des résultats}

Comme nous l'avons souligné dans la section 3, la méthode par appariement ne permet pas de prendre en compte l'hétérogénéité non observée. Or, il est possible que des variables puissent influer sur le fait de déposer un brevet et sur le salaire/la détention de stock-options sans que nous puissions les contrôler. Dans notre cas, cette hétérogénéité pourrait, par exemple, venir de la motivation des ingénieurs à innover. En effet, il semblerait que les inventeurs puissent avoir une forte motivation intrinsèque à la recherche, celle-ci n'est pas prise en compte dans nos estimations. L'existence de telle variable pourrait remettre en cause l'hypothèse d'indépendance conditionnelle. Afin de tester la robustesse de nos résultats, nous effectuons l' analyse de sensibilité développée par Rosenbaum (2002). Les résultats sont reportés dans le tableau $\mathrm{n}^{\circ} 1 \mathrm{D}$ pour les estimations concernant l'avantage salarial et dans le tableau $n^{\circ} 2 \mathrm{D}$ pour les estimations concernant la détention de stock-options. Puisque dans le premier cas, la variable d'intérêt est quantitative, le test utilisé est le test de Wilcoxon alors que dans le second cas, puisque la variable est binaire, nous utilisons le test de MantelHaenszel $^{7}$. L'interprétation est la même pour les deux tests.

Le tableau 1D montre que pour l'ensemble des ingénieurs le seuil critique à partir duquel les résultats des estimations de l'avantage salarial peuvent être contestables est de 1,15. En d'autres termes, à un changement de $15 \%$ dans le rapport de chance de recevoir le traitement pourrait rendre les estimations biaisées à cause des caractéristiques non observables. Par ailleurs, il semble que les estimations concernant les docteurs et les

\footnotetext{
${ }^{7}$ Ce test a été effectué à partir de la commande mhbounds développé par Becker et Caliendo (2007). Ce test ne peut être effectué qu'après un appariement par le plus proche voisin sans replacement.
} 
ingénieurs n'ayant pas connu de mobilité soient plus robustes à l'hétérogénéité non observée. En effet, les résultats risquent d'être biaisés à partir d'un seuil de 1,25. Par contre, les résultats concernant les ingénieurs travaillant à l'étranger ne passent pas le test d'hétérogénéité. Ce résultat est non surprenant puisque les résultats de l'ATT sont non significatifs pour cet souséchantillon.

Concernant les estimations se référant à la détention de stock-options, les résultats apparaissent très sensibles à l'hétérogénéité non observée pour les ingénieurs travaillant en France quelque soit l'échantillon considéré. Les résultats concernant les stock-options sont donc à prendre avec précaution. Par contre, les résultats concernant le sous-échantillon des expatriés sont fortement robustes à l'hétérogénéité, le seuil étant de 1,85.

Il est important de comprendre que l'analyse de sensibilité montre uniquement comment les biais peuvent modifier l'estimation. Mais, elle ne permet pas de déterminer si ces biais existent (Aakvik, 2001). Par exemple, les résultats du tableau montrent uniquement que l'intervalle de confiance du gain salarial pourrait inclure zéro si une variable non observable faisait varier le rapport de cotes d'être traité entre les deux groupes d'un facteur de 1,15 pour l'ensemble des ingénieurs et de 1,25 pour les docteurs. Dans la littérature, il n'est pas fait référence à un niveau de seuil conseillé. Rosenbaum (2002) souligne, tout de même, que le test correspond au "pire scénario".

\section{Conclusion}

Dans cet article, nous nous sommes intéressés aux incitations monétaires à l'innovation au sein des entreprises. Tout d'abord, les résultats révèlent un gain salarial positif significatif pour les inventeurs, cet avantage est d'autant plus important si l'ingénieur est docteur. Il apparaît également un rendement salarial plus élevé pour les inventeurs ayant connu une mobilité par rapport à ceux n'ayant pas connu de mobilité ces cinq dernières années. Ce résultat pourrait suggérer que les entreprises sont prêtes à payer les connaissances acquises par les ingénieurs au sein des autres entreprises (Dahl, 2002). Les incitations apparaissent aussi différentes selon la nature de l'entreprise de l'inventeur. En effet, les incitations monétaires sont beaucoup plus importantes dans les petites entreprises et plus particulièrement au sein des PME de haute technologie que dans les grandes entreprises. 
D'autre part, l'utilisation de stock-options comme moyen de compensation des inventeurs apparaît peu utilisé par les entreprises françaises. Il semble néanmoins que les inventeurs n'ayant pas changé d'entreprise ont plus de chance de détenir des stock-options que les ingénieurs non-inventeurs, ce qui pourrait suggérer que les entreprises utilisent les stockoptions comme incitation pour retenir les inventeurs. Ce résultat est tout de même à prendre avec précaution puisqu'il ne satisfait pas le test d'hétérogénéité. Enfin, l'analyse des ingénieurs expatriés révèle un système de compensation des inventeurs à l'étranger très différent au système français. L'utilisation de stock-option comme incitations pour les inventeurs apparaît plus développé dans les entreprises étrangères que dans les entreprises françaises. 
Annexe A: Score de propension

Figure $\mathrm{n}^{\circ} 1 \mathrm{~A}$ : Score de propension pour l'ensemble des ingénieurs

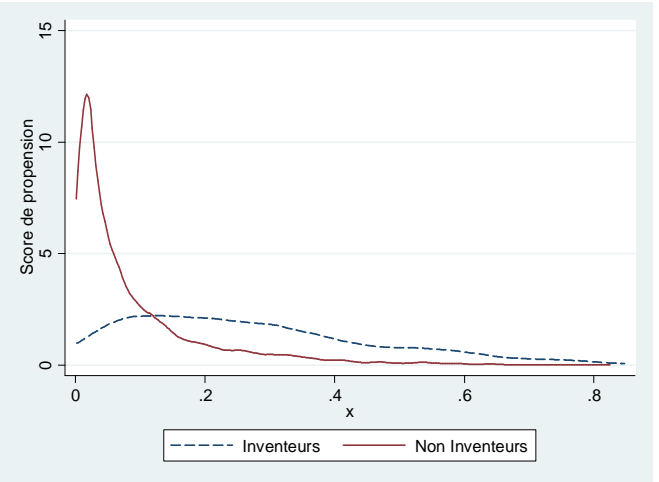

Figure $\mathrm{n}^{\circ} 2 \mathrm{~A}$ : Score de propension : Ingénieurs n'ayant pas connu de mobilité inter-firme

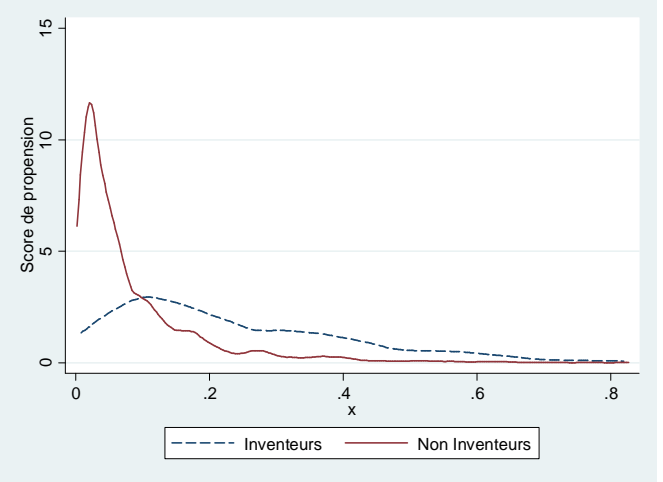

Figure $n^{\circ} 3 \mathrm{~A}$ : Score de propension : Ingénieurs ayant connu une mobilité inter-firme

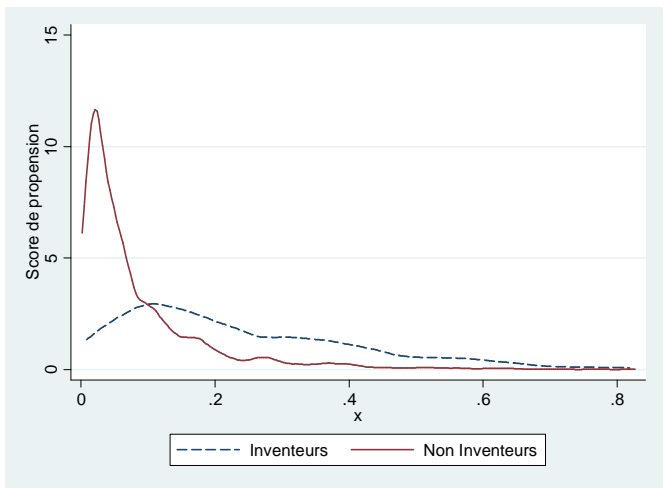


Tableau $\mathrm{n}^{\circ} 1 \mathrm{~A}$ : Tests de la propriété d'"équilibrage"

\begin{tabular}{|c|c|c|c|c|c|c|}
\hline & \multicolumn{2}{|c|}{$\begin{array}{c}\text { Ensemble } \\
\text { des ingénieurs }\end{array}$} & \multicolumn{2}{|c|}{$\begin{array}{c}\text { Ingénieurs } \\
\text { sans mobilité }\end{array}$} & \multicolumn{2}{|c|}{$\begin{array}{c}\text { Ingénieurs avec } \\
\text { mobilité }\end{array}$} \\
\hline & $\begin{array}{l}\text { Sign. t- } \\
\text { test }\end{array}$ & $\begin{array}{l}\text { Diff. } \\
\text { stand. }\end{array}$ & $\begin{array}{l}\text { Sign.t- } \\
\text { test }\end{array}$ & $\begin{array}{l}\text { Diff. } \\
\text { stand. }\end{array}$ & $\begin{array}{l}\text { Sign.t- } \\
\text { test }\end{array}$ & $\begin{array}{l}\text { Diff. } \\
\text { stand. }\end{array}$ \\
\hline \multicolumn{7}{|l|}{ Caractéristiques individuelles } \\
\hline Genre & Ns & $<20$ & Ns & $<20$ & Ns & $<20$ \\
\hline Doctorat & Ns & $<20$ & Ns & $<20$ & Ns & $<20$ \\
\hline $\begin{array}{l}\text { Ancienneté sur le marché du travail } \\
\text { Ancienneté au carré }\end{array}$ & $\begin{array}{l}\text { Ns } \\
\text { Ns }\end{array}$ & $<20$ & $\begin{array}{c}* \\
\mathrm{Ns}\end{array}$ & $<20$ & Ns & $<20$ \\
\hline Activité dominante de l'ingénieur & $*$ & $<20$ & Ns & $<20$ & $*$ & $<20$ \\
\hline Participation à un projet de l'UE & Ns & $<20$ & Ns & $<20$ & Ns & $<20$ \\
\hline \multicolumn{7}{|l|}{ Caractéristiques de l'entreprise } \\
\hline Secteur de l'entreprise & Ns & $<20$ & Ns & $<20$ & Ns & $<20$ \\
\hline Taille de l'entreprise & Ns & $<20$ & Ns & $<20$ & Ns & $<20$ \\
\hline $\begin{array}{l}\text { Politique de veille concurrentielle } \\
\text { stratégique }\end{array}$ & Ns & $<20$ & Ns & $<20$ & Ns & $<20$ \\
\hline Dépenses de R\&D de l'entreprise & Ns & $<20$ & Ns & $<20$ & Ns & $<20$ \\
\hline Partenariat avec des universités & Ns & $<20$ & Ns & $<20$ & Ns & $<20$ \\
\hline
\end{tabular}

Note: *** sign. à $1 \%$, ** sign. à 5\%, * sign à $10 \%$. 
Annexe B : Résultats des Probit

Tableau $\mathrm{n}^{\circ} 1 \mathrm{~B}$ : Résultats des probit estimation du score de propension

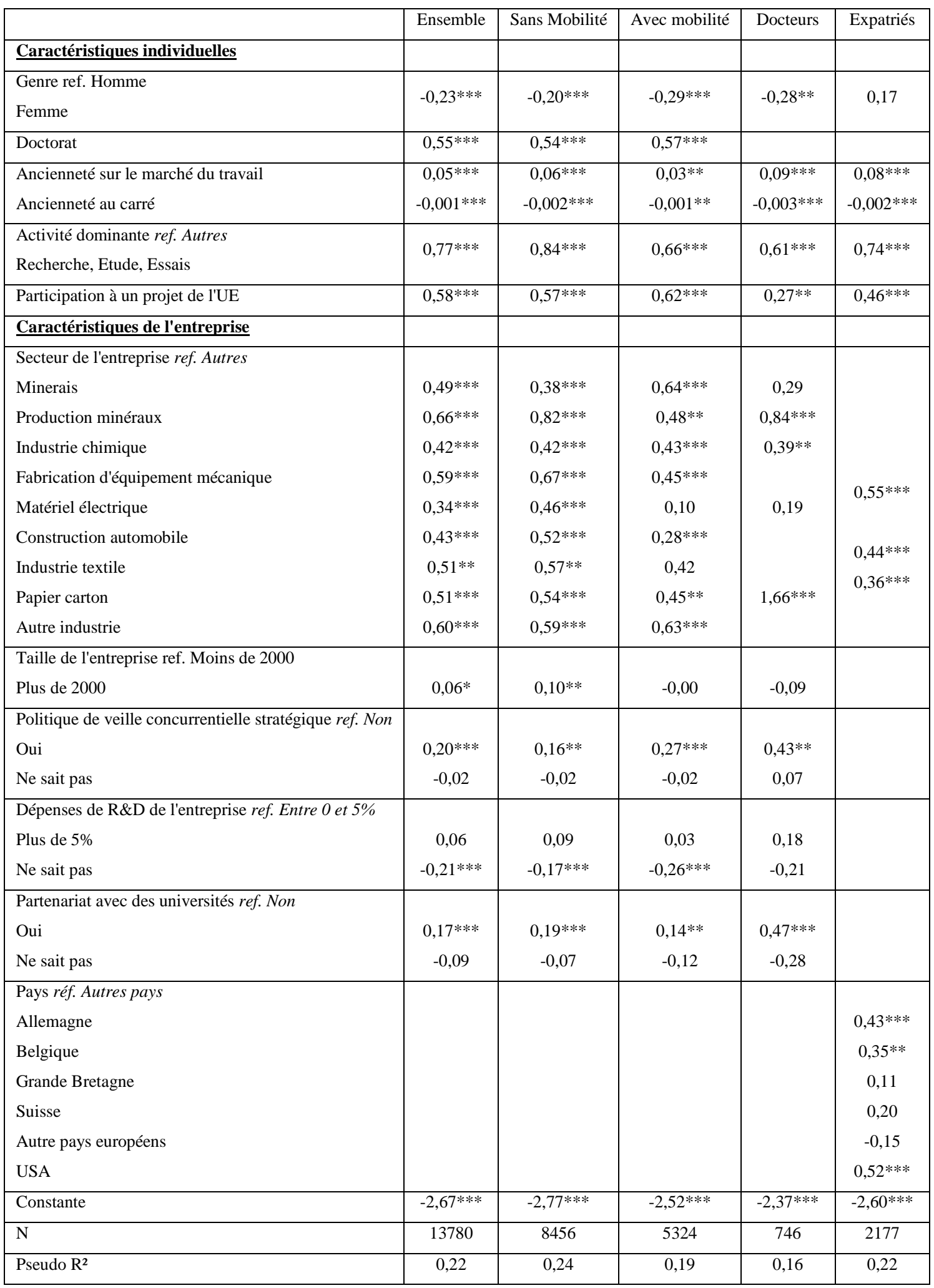

Note: *** sign. à $1 \%$, ** sign. à 5\%, * sign à $10 \%$. 
Annexe C : Résultats des MCO

Tableau $\mathrm{n}^{\circ} 1 \mathrm{C}$ : Résultats des régressions par $\mathrm{MCO}$

\begin{tabular}{|c|c|c|c|c|c|}
\hline Variables dépendante: $\log$ du salaire annuel & Ensemble & $\begin{array}{l}\text { ingénieurs } \\
\text { sans mobilité }\end{array}$ & ingénieurs avec mobilité & Docteurs & Expatriés \\
\hline Brevet & $\underline{\mathbf{0 , 0 2 9}} * * *$ & $\underline{\mathbf{0 , 0 2 7} * * *}$ & $\underline{\mathbf{0 , 0 3 6} * * *}$ & $\underline{\mathbf{0 , 0 5 9} * * *}$ & $\underline{-0,01}$ \\
\hline \multicolumn{6}{|l|}{ Caractéristiques individuelles } \\
\hline $\begin{array}{c}\text { Genre ref. Homme } \\
\text { Femme }\end{array}$ & $-0,08 * * *$ & $-0,07 * * *$ & $-0,11 * * *$ & $-0,04 *$ & $-0,10$ \\
\hline Doctorat & $0,02 * *$ & $0,03 *$ & 0,02 & & $0,08 * * *$ \\
\hline $\begin{array}{l}\text { Ancienneté sur le marché du travail } \\
\text { Ancienneté au carré }\end{array}$ & $\begin{array}{l}0,06 * * * \\
-0,001 * * *\end{array}$ & $\begin{array}{l}0,06 * * * \\
-0,001 * * *\end{array}$ & $\begin{array}{l}0,07 * * * \\
-0,001 * * *\end{array}$ & $\begin{array}{l}0,05 * * * \\
-0,000 * * *\end{array}$ & $\begin{array}{l}0,08 * * * \\
-0,001 * * *\end{array}$ \\
\hline $\begin{array}{c}\text { Activité dominante ref. Autres } \\
\text { Recherche, Etude, Essais }\end{array}$ & $-0,08 * * *$ & $-0,07 * * *$ & $-0,9 * * *$ & $-0,11 * * *$ & $-0,10 * * *$ \\
\hline Participation à un projet de l'UE & 0,01 & 0,01 & 0,01 & 0,02 & $-0,05^{*}$ \\
\hline \multicolumn{6}{|l|}{ Caractéristiques de l'entreprise } \\
\hline $\begin{array}{c}\text { Secteur de l'entreprise ref. Autres } \\
\text { Minerais } \\
\text { Production minéraux } \\
\text { Industrie chimique } \\
\text { Fabrication d'équipement mécanique } \\
\text { Matériel électrique } \\
\text { Construction automobile } \\
\text { Industrie textile } \\
\text { Papier carton } \\
\text { Autre industrie }\end{array}$ & $\begin{array}{c}0,02 \\
0,10 * * * \\
0,06 * * * \\
-0,04 * * * \\
-0,02 * * \\
0,01 * * \\
-0,01 \\
0,03 \\
-0,03 * *\end{array}$ & $\begin{array}{c}0,01 \\
0,11 * * * \\
0,07 * * * \\
-0,04 * * * \\
-0,02 * * \\
0,02 * \\
-0,02 \\
0,01 \\
-0,03 * *\end{array}$ & $\begin{array}{c}0,04 \\
0,08 * * * \\
0,04 * * \\
-0,04 * * \\
-0,01 \\
0,01 \\
0,00 \\
0,05 * \\
-0,02\end{array}$ & $\begin{array}{c}0,04 \\
0,09 * \\
0,10 * * * \\
-0,01 \\
-0,06 \\
0,05 \\
0,06 \\
0,09 \\
0,01\end{array}$ & $\begin{array}{l}-0,02 \\
-0,04 *\end{array}$ \\
\hline $\begin{array}{c}\text { Taille de l'entreprise ref. moins de } 2000 \\
\text { Plus de } 2000\end{array}$ & $0,07 * * *$ & $0,07 * * *$ & $0,08 * * *$ & $0,07 * * *$ & \\
\hline $\begin{array}{c}\text { Pays réf. Autres pays } \\
\text { Allemagne } \\
\text { Belgique } \\
\text { Grande Bretagne } \\
\text { Suisse } \\
\text { Autres pays d'europe } \\
\text { USA }\end{array}$ & & & & & $\begin{array}{c}0,08 * * * \\
-0,055 \\
-0,04 \\
0,17 * * * \\
-0,06 * * * \\
0,31 * * *\end{array}$ \\
\hline Constante & $10,32 * * *$ & $10,34 * * *$ & $10,28 * * *$ & $10,42 * * *$ & $10,5 * * *$ \\
\hline $\mathrm{N}$ & 13780 & 8456 & 5324 & 746 & 2177 \\
\hline $\mathrm{R}^{2}$ ajusté & 0,62 & 0,65 & 0,56 & 0,60 & 0,55 \\
\hline
\end{tabular}

Note: *** sign. à $1 \%$, ** sign. à $5 \%$, * sign à $10 \%$. 
Annexe D : Robustesse des résultats

Tableau $n^{\circ} 1 \mathrm{D}:$ Test de Rosenbaum ${ }^{8}$

\begin{tabular}{|c|c|c|c|c|c|}
\hline & Ensemble des ingénieurs & Ingénieurs sans mobilité & Ingénieurs avec mobilité & Docteurs & Expatriés \\
\hline 1 & 0,000394 & 0,004055 & 0,000429 & 0,00261 & 0,087653 \\
\hline 1,05 & 0,005604 & 0,023497 & 0,002168 & 0,007187 & $\mathbf{0 , 1 5 5 2 7}$ \\
\hline 1,1 & 0,039625 & 0,087373 & 0,008289 & 0,01701 & 0,245326 \\
\hline 1,15 & $\mathbf{0 , 1 5 6 3 4 4}$ & $\mathbf{0 , 2 2 4 6 6 7}$ & 0,02495 & 0,03534 & 0,352253 \\
\hline 1,20 & 0,383483 & 0,427845 & 0,061155 & 0,065603 & 0,467087 \\
\hline 1,25 & 0,650865 & 0,644033 & $\mathbf{0 , 1 2 5 6 6 9}$ & $\underline{\mathbf{0 , 1 1 0 4 7 4}}$ & 0,580005 \\
\hline 1,30 & 0,851982 & 0,815648 & 0,222098 & 0,170971 & 0,682723 \\
\hline 1,35 & 0,953313 & 0,920565 & 0,345371 & 0,245935 & 0,769924 \\
\hline 1,40 & 0,98894 & 0,971335 & 0,482427 & 0,332095 & 0,839533 \\
\hline 1,45 & 0,997999 & 0,99125 & 0,616879 & 0,424671 & 0,89212 \\
\hline 1,50 & 0,999718 & 0,997715 & 0,734723 & 0,5183 & 0,92993 \\
\hline 1,55 & 0,999969 & 0,999483 & 0,828023 & 0,60796 & 0,955928 \\
\hline 1,60 & 0,999997 & 0,999898 & 0,895389 & 0,689684 & 0,973098 \\
\hline 1,65 & 1 & 0,999982 & 0,940124 & 0,760906 & 0,984029 \\
\hline 1,70 & 1 & 0,999997 & 0,967649 & 0,820494 & 0,990761 \\
\hline 1,75 & 1 & 1 & 0,983445 & 0,868524 & 0,994782 \\
\hline 1,80 & 1 & 1 & 0,99195 & 0,90594 & 0,997118 \\
\hline 1,85 & 1 & 1 & 0,996268 & 0,93419 & 0,998441 \\
\hline 1,90 & 1 & 1 & 0,998346 & 0,954914 & 0,999173 \\
\hline 1,95 & 1 & 1 & 0,999297 & 0,969718 & 0,999569 \\
\hline 2,00 & 1 & 1 & 0,999713 & 0,980039 & 0,999779 \\
\hline
\end{tabular}

Tableau n $^{\circ}$ 2D: Test de Mantel-Haenszel ${ }^{9}$

\begin{tabular}{|c|c|c|c|c|}
\hline & Ensemble des ingénieurs & Ingénieurs sans mobilité & Ingénieurs avec mobilité & Expatriés \\
\hline 1 & 0,024216 & 0,142897 & 0,557636 & 0,000241 \\
\hline 1,05 & 0,043186 & 0,194259 & 0,490781 & 0,000473 \\
\hline 1,1 & 0,070956 & 0,252413 & 0,427219 & 0,000879 \\
\hline 1,15 & $\mathbf{0 , 1 0 8 5 7 1}$ & 0,315433 & 0,368151 & 0,001541 \\
\hline 1,20 & 0,156222 & 0,381237 & 0,314314 & 0,002568 \\
\hline 1,25 & 0,213138 & 0,447747 & 0,26607 & 0,004089 \\
\hline 1,30 & 0,27768 & 0,513074 & 0,223477 & 0,006252 \\
\hline 1,35 & 0,347577 & 0,519505 & 0,186364 & 0,009219 \\
\hline 1,40 & 0,420227 & 0,459087 & 0,154399 & 0,013161 \\
\hline 1,45 & 0,49302 & 0,401647 & 0,127152 & 0,018248 \\
\hline 1,50 & 0,512498 & 0,348068 & 0,104139 & 0,024645 \\
\hline 1,55 & 0,444377 & 0,29894 & 0,084862 & 0,032501 \\
\hline 1,60 & 0,37992 & 0,254583 & 0,068834 & 0,041945 \\
\hline 1,65 & 0,320442 & 0,215091 & 0,055597 & 0,053078 \\
\hline 1,70 & 0,266797 & 0,180374 & 0,044731 & 0,06597 \\
\hline 1,75 & 0,21941 & 0,150207 & 0,035859 & 0,080661 \\
\hline 1,80 & 0,178338 & 0,124267 & 0,028652 & 0,097151 \\
\hline 1,85 & 0,143357 & 0,102177 & 0,022823 & $\mathbf{0 , 1 1 5 4 1}$ \\
\hline 1,90 & 0,114036 & 0,083533 & 0,018129 & 0,135375 \\
\hline 1,95 & 0,089818 & 0,067923 & 0,014363 & 0,15695 \\
\hline 2,00 & 0,070086 & 0,054951 & 0,011352 & 0,180017 \\
\hline
\end{tabular}

${ }^{8}$ Ce test a été effectué avec la commande rbounds sous Stata. Ce test ne peut être effectué qu'après l'appariement par le plus proche voisin.

${ }^{9}$ Ce test a été effectué à partir de la commande mhbounds développé par Becker et Caliendo. Ce test ne peut être effectué qu'après un appariement par le plus proche voisin sans replacement. 


\section{Bibliographie}

Aakvik, A., (2001), "Bounding a matching estimator: the case of a norwegian training program", Oxford Bulletin of Economics and Statistics, vol.1, n63, pp.115-143.

Amabile T. (1996), Creativity in Context: Update to the Social Psychology of Creativity, Boulder, CO: Westview Press.

Andreosso-O'Callaghan, M.B. (2000), "Taxation and Innovation in the European Union", Korean Journal of EU Studies, vol.5, n¹, pp.121-145.

Backer G.P., Jensen M.C., Murphy K.J. (1988), "Compensation and Incentives: Practice vs. Theory", The Journal of Finance, vol.43, n³, pp.593-616.

Balkin D.B., Gomez-Mejia L.R. (1984), "Determinants of R and D compensation strategies in the high tech industry", Personnel Psychology, vol.37, pp.635-650.

Becker S.O., Caliendo M. (2007), "Sensitivity analysis for average treatment effects", The Stata Journal, vol.7, n 1 , pp.71-83.

Bénabou R., Tirole J. (2003), "Intrinsic and Extrinsic Motivation", Review of Economic Studies, vol.70, $\mathrm{n}^{\circ} 3, \mathrm{pp} .489-520$.

Bonnard C., Bourdon J., Paul J.J. (2011), "Travailler dans la recherche privée au sortir d'une école d'ingénieur: est-ce la bonne stratégie?", Revue d'économie industrielle, à parâtre.

Brodaty T., Crépon B., Fougère D. (2007), "Les méthodes micro-économétriques d'évaluation et leurs applications aux politiques actives de l'emploi", Economie et Prévision, vol.1, n ${ }^{\circ}$ 177, pp.93-118.

Burhop C., Lübbers T. (2010), "Incentives and innovation? R\&D management in Germany's chemical and electrical engineering industries around 1900", Explorations in Economic History, vol.47, pp.100-111.

Caliendo M., Kopeinig S. (2008), "Some practical Guidance for the Implementation of propensity score matching", Journal of Economic Surveys, vol.22, n¹, pp.31-72. 
Cohen W., Sauerman H. (2008), "What makes them tick? Employee Motives and Industrial Innovation", NBER Working Paper.

Crépon B., Duguet E., Mairesse J. (1998), "Research, innovation, and productivity: an econometric analysis at the firm level", Economics of Innovation and New Technology, vol.7, $\mathrm{n}^{\circ} 2, \mathrm{pp} .115-158$.

Dahl M. (2002),"Embedded knowledge flows through labor mobility in regional clusters in Denmark", Presented at DRUID's New Economy Conference, Copenhagen, June 2002.

Dasgupta P., David P. (1994), "Toward a New Economics of Science", Research Policy, vol.23, pp.487-521.

Deci E.L. (1975), Intrinsic motivation. New York: Plenum.

Dupuy A., Smits W. (2009), "How large is the compensating wage differential for R\&D workers?" , Discussion Papers 4194, Institute for the Study of Labor.

Ederer F., Manso G. (2009), "Is pay-for performance detrimental to innovation?", Working Paper, MIT.

Eisenhardt K.M. (1989), "Agency theory: an assessment review", The Academy of Management Review, vol.14, n 1 pp.57-74.

Fama E. (1980), "Agency problems and the theory of the firm", Journal Political Economy, vol.88, pp. 288-307.

Francis B., Hasan I., Sharma Z. (2009), "Do incentives create innovation? Evidence from CEO compensation contracts", Working Paper, Rensselaer Polytechnic Institute.

Frey B.S. (2007), "Awards as compensation", European Management Review, vol.4, pp.6-14.

Frey B.S., Jegen R. (2001), "Motivation Crowding Theory: a survey of empirical evidence", Journal of Economics Survey, vol.15, n ${ }^{\circ}$, pp.589-611.

Frey B.S., Osterloh M. (2002), Successful management by motivation. Balancing Intrinsic and Extrinsic Incentives, New York: Springer. 
Frölich M. (2007). "Propensity score matching without conditional independence assumptionwith an application to the gender wage gap in the United Kingdom", Econometrics Journal, vol.10, $\mathrm{n}^{\circ} 2$, pp. 359-407.

George G., Zahra S.A., Wood D.R. (2002), "The effects of business-university alliances on innovative output and financial performance: a study of publicly traded biotechnology companies", Journal of Business Venturing, vol.17, pp.577-609.

Gersbach H. , Schmultzler A. (2003), "Endogenous technical spillovers: causes and consequences", Journal of Economics and Management Strategy, vol.12, n², pp.179-205.

Gibbs M., Levenson A. (2002), The economic approach to personnel research, dans S. Grossbard-Schechtman et C. Clague (eds.), The Expansion of Economics: Toward a More Inclusive Social Science, M.E. Sharpe: Armonk NY, pp.99-139.

Giuri et al. (2007), "Inventors and invention processes in Europe: Results from the PatVal-EU survey", Research policy, n³6, pp. 1107-1127.

Griliches Z. (1984), R\&D, Patents and Productivity, Chicago, University of Chicago Press.

Hall B.J., Murphy K.J. (2003), "The Trouble with Stock Options", The Journal of Economic Perspectives, vol.17, n³, pp.49-70.

Harhoff D., Hoisl K. (2007), "Institutionalized incentives for ingenuity-patent value and the German employees' inventions Act", Research Policy, vol.36, n8, pp.1143-1162.

Heckman J., Ichimura H., Todd P. (1997), "Matching as an Econometric Evaluation Estimator: Evidence from Evaluating a Job training Program", Review of Economic Studies, vol.64, pp. 605-654.

Heckman J., Ichimura H., Todd P. (1998), "Matching as an Econometric Evaluation Estimator", Review of Economic Studies, vol.65, pp. 261-294.

Hoisl K. (2007), "A closer look at inventive output, the role of age and career paths", Discussion Paper. 
Hoisl K. (2007), "Tracing mobile inventors, the causality between inventor mobility and inventor productivity", Research Policy, vol. 36, pp.619-636.

Holmstrom, T.P. (1979), "Moral hazard and observability" , Bell Journal of Economics, vol.10, pp.74-91.

Honig-Haftel S., Martin L. (1993), "The Effectiveness of Reward Systems on Innovative Output: An Empirical Analysis", Small Business Economics, vol.5, n²4, pp.261-269.

Jones C.I. (2005), "Growth and Ideas", dans Aghion P. et Durlauf S.N. eds. Handbook of Economic Growth, North-Holland.

Kim B. , Oh H. (2002), "An effective R\&D performance measurement: survey of Korean R\&D researchers", Omega, vol.30, pp.19-31.

Kreps D.M. (1997), "Intrinsic Motivation and Extrinsic Incentives", The American Economic Review, vol.87, $\mathrm{n}^{\circ} 2, \mathrm{pp} .359-364$.

Kochanski J., Ledford G. (2001), "How to keep me: Retaining technical professionals", Research-Technology Management, p.31-38.

Lacetera N., Zirulia L. (2008), "Knowledge spillovers, Competition, and Taste for Science in a Model of R\&D Incentive Provision", Working Paper.

Lach S., Schankerman M. (2008), "Incentives and invention in universities", The Rand Journal of Economics, vol.39, n², pp.403-433.

Lawler E.E. (2003), Reward Systems in Knowledge-based Organizations. In Jackson S.E., Hitt M.A. et DeNisi A.S. (Eds.), Managing knowledge for sustained competitive advantage: Designing strategies for effective human resource management (pp. 274-302). San Francisco: Jossey-Bass.

Leuven E, Sianesi B. (2003), "PSMATCH2: Stata module to perform full Mahalanobis and propensity score matching, common support graphing, and covariate imbalance testing". http://ideas.repec.org/c/boc/bocode/s432001.html. 
Lerner J., Wulf J. (2007), "Innovation and incentives: Evidence from corporate R\&D", the Review of Economics and Statistics, vol.89, n 4 , pp.634-644.

Levin S.G., Stephan P.E (1991), "Research productivity over the cycle life: evidence for academic scientists", The American Economic Review, vol.81, n¹, pp. 114-132.

Liebeskind J.P. (1996), "Knowledge, strategy, and the theory of the firm", Strategic Management Journal, vol.17, pp.93-107.

Mariani M., Romanelli M. (2007), "'Stacking" and "picking" inventions: the patenting behavior of European inventors", Research Policy, vol.36, pp.1128-1142.

Mairesse J., Mohnen P. (2005), "The importance of R\&D for innovation: a reassessment using French survey data", The Journal of Technology Transfer, vol.30 (1-2), pp.183-197.

McMillan G.S. (2009), "Gender differences in patenting activity: an examination of the US biotechnology industry", Scientometrics, vol.80, n³, pp.683-691.

Mehran H., Yermack D. (1999), "Compensation and Top Management Turnover", Working paper, New York University.

Mendez A. (2006), "Vers une globalisation de la gestion des ressources humaines?", Cahiers Français, n $^{\circ} 333$, pp.38-43.

Merton R. (1973), The Sociology of Science, University of Chicago Press.

Moen J. (2005), "Is mobility of technical personnel a source of R\&D spillovers?", Journal of Labor Economics, vol.23, n¹, pp.81-114.

Observatoire de la Propriété intellectuelle (2008), Enquête sur la rémunération des inventeurs salariés, INPI.

Osterloh M. (2005), Human Resources Management and Knowledge Creation, dans Nonaka I. et Kazuo I. eds., Handbook of Knowledge Creation, Oxford University Press.

Oyer P., Schaefer S. (2005), "Why do some firms give stock options to all employees?: An empirical examination of alternative theories", Journal of Financial Economics, vol.76, n 1 , pp.99-133. 
Pakes A., Nitzan S. (1983), "Optimum contracts for research personnel, research employment, and the establishment of "rival" enterprises", Journal of Labor Economics, vol.1, pp.345-365.

Prendergast C. (1999), "The provision of incentives in Firms", Journal of economic literature, vol.37, $\mathrm{n}^{\circ} 1, \mathrm{pp} .7-63$.

Radhakrishnan S., Ronen J. (1999), "Job challenge as a motivator in a principal-agent setting", European Journal of Operational Research, vol.115, pp.138-157.

Roach M., Sauerman H. (2010), "A taste for science? PhD scientists' academic orientation and self selection into research careers in industry", Research Policy, vol.39, n³, pp.422-434.

Rosenbaum P., Rubin D. (1983), "The central role of the propensity score in observational studies for causal effects", Biometrika, vol.70, pp.41-55.

Rosenbaum P. (2002),Observational studies. New York: Springer-Verlag.

Rossman J. (1931), "The Motives of Inventors", The quarterly Journal of Economics, vol.45, $\mathrm{n}^{\circ} 3$, pp. 522-528.

Rothaermel F.T., Hess A.M. (2007), "Building dynamic capabilities: innovation driven by individual, firm, and network-level effects", Organization Science, vol.18, n6, pp.898-921.

Salop S.C. (1979), "A model of the natural rate of unemployment", American Economic Review, vol.69, pp.117-125.

Shapiro C., Stiglitz J.E. (1984), "Equilibrium Unemployment as a Worker Discipline Device", The American Economic Review, vol.74, n³, pp.433-444.

Smith J., Todd P. (2005), "Does Matching Overcome LaLonde's Critique of Nonexperimental Estimators?", Journal of Econometrics, vol.125, n¹-2, pp. 305-353.

Stern S. (2004), "Do scientists pay to be scientists?", Management Science, vol.50, n6, pp. 835-853.

Toivanen O., Väänänen L. (2010), "Returns to Inventors", Discussion Paper n³09. 
Tosi, H.L., Gomez-Mejia, L.R. (1989), "The decoupling of CEO pay and performance: An agency theory perspective", Administrative Science Quarterly, vol.34, pp.169-189.

Titus M.A. (2007), "Detecting selection bias, using propensity score matching, an estimating treatment effects: An application to the private retunes to a Master's Degree", Research in Higher Education, vol.48, ${ }^{\circ} 7$, pp.487-521.

Traijtenberg M. (2005), Recombinant ideas: the mobility of inventors and the productivity of research, CEPR-Conference, Munich, May 26-28, 2005.

Whittington K., Smith-Doerr L. (2005), "Gender and commercial science : women's patenting in the life sciences", Journal of Technology Transfer, vol.30, pp.355-370.

Zenger T.R. (1994), "Explaining Organizational Diseconomies of Scale in R\&D: Agency Problems and the Allocation of Engineering Talent, Ideas, and Effort by Firm Size", Management Science, vol.40, nº, pp.708-729.

Zenger T.R., Lazzarini S.G. (2004), "Compensating for innovation: Do small firms offer higher-powered incentives that lure talent and motive effort?", Managerial and Decision Economics, vol.25, pp.329-345. 